\title{
Development of a detailed chemical mechanism (MCMv3.1) for the atmospheric oxidation of aromatic hydrocarbons
}

\author{
C. Bloss ${ }^{1}$, V. Wagner ${ }^{1}$, M. E. Jenkin ${ }^{2}$, R. Volkamer ${ }^{3, *}$, W. J. Bloss ${ }^{1}$, J. D. Lee ${ }^{1, * *}$, D. E. Heard ${ }^{1}$, K. Wirtz ${ }^{4}$, \\ M. Martin-Reviejo ${ }^{4}$, G. Rea ${ }^{5}$, J. C. Wenger ${ }^{5}$, and M. J. Pilling ${ }^{1}$ \\ ${ }^{1}$ School of Chemistry, University of Leeds, Leeds LS2 9JT, UK \\ ${ }^{2}$ Imperial College London, Silwood Park, Ascot, Berkshire SL5 7PY, UK \\ ${ }^{3}$ Institut für Umweltphysik, University of Heidelberg, INF 229, 69120 Heidelberg, Germany \\ ${ }^{4}$ Centro de Estudios Ambientales del Mediterraneo, C. Charles R. Darwin 14, 46980 Paterna, Spain \\ ${ }^{5}$ Department of Chemistry, National University of Ireland, University College Cork, Cork, Ireland \\ *now at: Department of Earth, Atmospheric, and Planetary Sciences, Massachusetts Institute of Technology, 77 \\ Massachusetts Ave., Cambridge, MA 02139-4307, USA \\ ${ }^{* *}$ now at: Department of Chemistry, University of York, York, YO10 5DD, UK
}

Received: 27 July 2004 - Published in Atmos. Chem. Phys. Discuss.: 24 September 2004

Revised: 13 December 2004 - Accepted: 5 February 2005 - Published: 1 March 2005

\begin{abstract}
The Master Chemical Mechanism has been updated from MCMv3 to MCMv3.1 in order to take into account recent improvements in the understanding of aromatic photo-oxidation. Newly available kinetic and product data from the literature have been incorporated into the mechanism. In particular, the degradation mechanisms for hydroxyarenes have been revised following the observation of high yields of ring-retained products, and product studies of aromatic oxidation under relatively low $\mathrm{NO}_{\mathrm{x}}$ conditions have provided new information on the branching ratios to first generation products. Experiments have been carried out at the European Photoreactor (EUPHORE) to investigate key subsets of the toluene system. These results have been used to test our understanding of toluene oxidation, and, where possible, refine the degradation mechanisms. The evaluation of MCMv3 and MCMv3.1 using data on benzene, toluene, $p$-xylene and 1,3,5-trimethylbenzene photosmog systems is described in a companion paper, and significant model shortcomings are identified. Ideas for additional modifications to the mechanisms, and for future experiments to further our knowledge of the details of aromatic photo-oxidation are discussed.
\end{abstract}

\section{Introduction}

The photo-oxidation of volatile organic compounds (VOC) in the presence of nitrogen oxides $\left(\mathrm{NO}_{\mathrm{x}}\right)$ leads to the for-

Correspondence to: M. J. Pilling

(m.j.pilling@leeds.ac.uk) mation of photochemical smog, and aromatic hydrocarbons have been found to contribute significantly to the production of ozone on a regional scale. In particular, toluene, which has high emissions and a comparatively high photochemical ozone creation potential, was identified as the VOC leading to the most ozone formation along an idealised trajectory, characteristic of a photochemical pollution episode in northwest Europe (Derwent et al., 1996).

Air quality models make use of detailed photochemical mechanisms to assess the importance of the emission of air pollutants on the formation of ozone. The Master Chemical Mechanism (MCM) is one such mechanism, and consists of a near-explicit representation of the degradation of a large number of emitted VOC, including aromatic compounds. It is constructed using kinetic and product data for elementary reactions involved in VOC oxidation (Jenkin et al., 1997, 2003; Saunders et al., 2003), and describes the formation of ozone and other secondary pollutants resulting from that oxidation. A good understanding of the oxidation mechanisms of aromatics in the atmosphere is necessary for reliable assessment of the impact of their emissions on air quality.

Until recently, knowledge of the detailed chemistry of aromatic photo-oxidation was fairly limited and early versions of MCM used highly simplified schemes to represent these degradations. As a result of the importance of aromatic compounds to regional air quality, experimental research in this field has been particularly active in recent years (e.g. Calvert et al., 2002 and references therein). The available information was used to construct detailed degradation mechanisms for 18 aromatic compounds in MCMv3 (Jenkin et al., 2003). However, Calvert et al. (2002) highlighted several

(C) 2005 Author(s). This work is licensed under a Creative Commons License. 


\section{MCMv3}

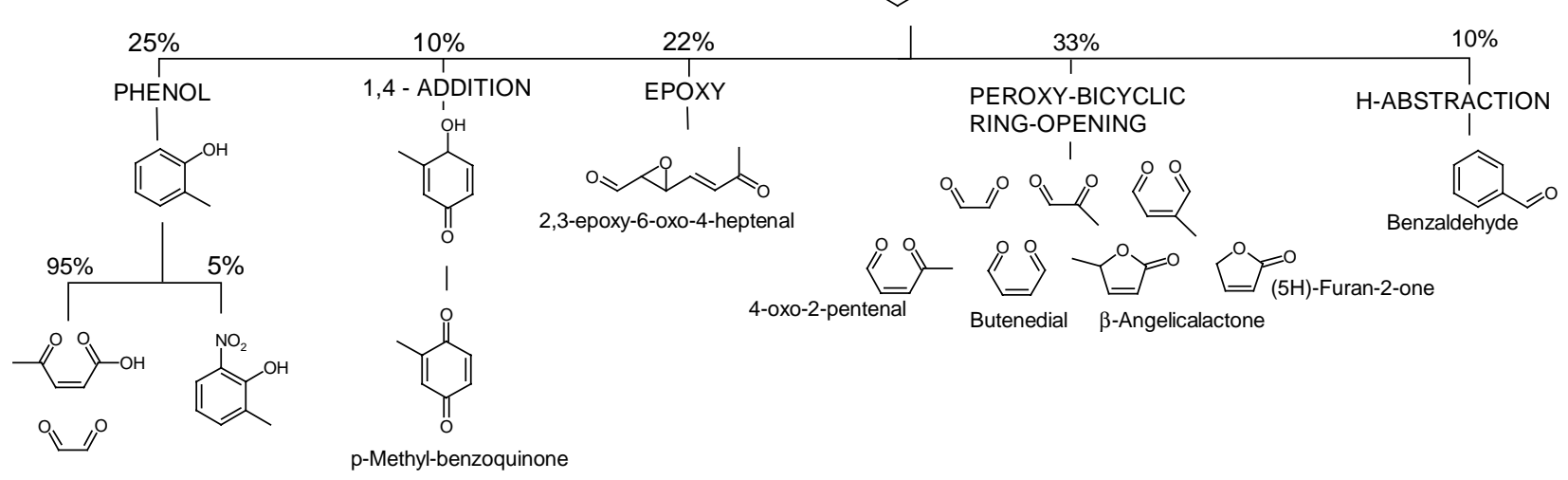

MCMv3.1

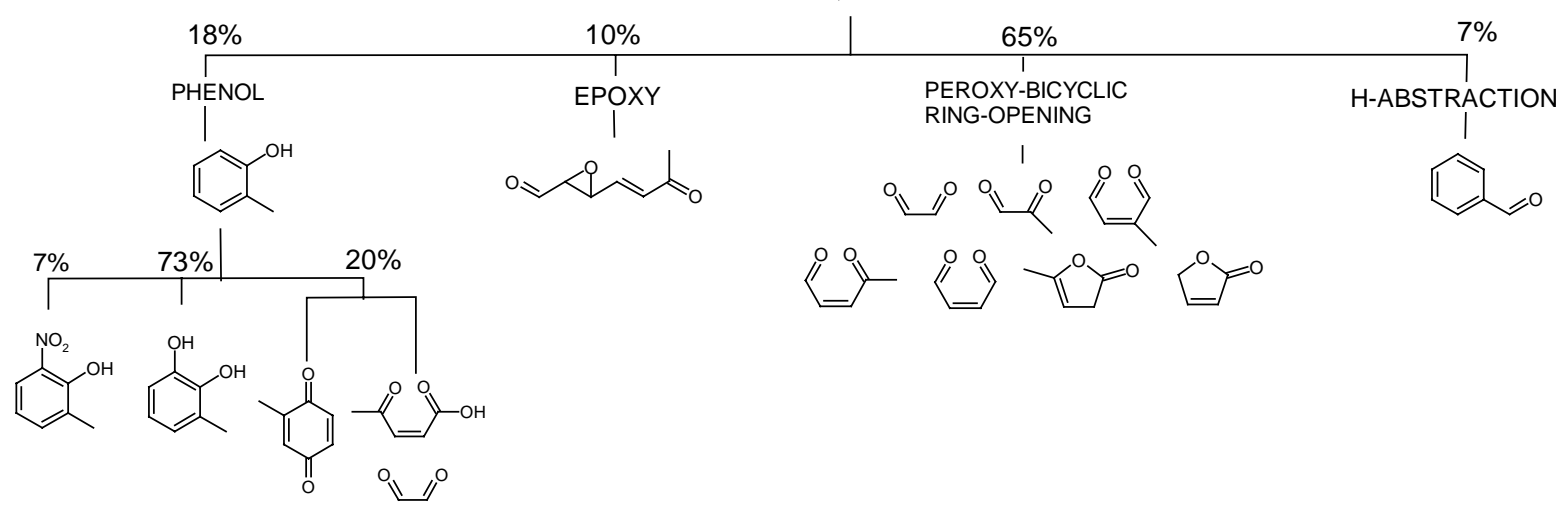

Fig. 1. Schematic representations of toluene oxidation mechanisms. Upper panel MCMv3, lower panel MCMv3.1.

uncertain aspects remaining in the understanding of aromatic oxidation chemistry, including: 1) product yields for reaction of $\mathrm{OH}$ with aromatic compounds, 2) mechanisms and products of the reactions of unsaturated dicarbonyls with atmospheric oxidising species, 3) gas phase photochemistry of ring-fragmentation products. Since that writing, further work has been carried out to address these issues. In particular a research project, EXACT, (Effects of the Oxidation of Aromatic Compounds in the Troposphere), was set up with the aim of improving and evaluating detailed chemical mechanisms for aromatic oxidation. Within that project, two experimental campaigns were carried out at EUPHORE (European Photoreactor); a comprehensive dataset on aromatic photosmog systems was obtained, and experiments were performed to test specific mechanistic details and provide a resource for further mechanism development. As toluene has high emissions and is the most intensively studied aromatic to date, it was chosen as the focus of the mechanism development work. Results on this system can be transferred to other aromatic hydrocarbons as the chemical mechanisms are expected to be similar.
The oxidation pathways of toluene implemented in MCMv3 are shown in the upper panel of Fig. 1. Habstraction from the methyl group leads to the formation of benzaldehyde as a first generation reaction product while the other channels involve sequential addition of $\mathrm{OH}$ and $\mathrm{O}_{2}$ to the aromatic ring. The well-established hydroxyarene product, $o$-cresol (2-methyl-phenol), is formed by elimination of $\mathrm{HO}_{2}$, and the $\mathrm{OH}$ initiated oxidation of cresol yields mainly the ring-opened products, glyoxal and 4-oxo-pent-2enoic acid. $p$-Methyl-benzoquinone has been observed as a product of toluene oxidation and in MCMv3 is a second generation product in the minor 1,4-addition route. The remaining pathways in MCMv3 stem from the chemistry of the methyl substituted 1,2-hydroxycyclohexadienyl peroxy radical. The furanones and $\gamma$-dicarbonyl compounds are proposed co-products of glyoxal and methylglyoxal formed as primary products from the decomposition of a peroxidebicyclic radical. Epoxy-type products have also been proposed as aromatic oxidation products (Bartolotti and Edney 1995), and support for their formation has been provided by product studies (Kwok et al., 1997; Yu and Jeffries, 1997). 
Table 1. Relative branching ratios assigned to $\mathrm{OH}$-initiated oxidation routes to first generation products in MCMv3.1. Assigned as for MCMv3 (Jenkin et al., 2003) updated as detailed in the text to take into account newly available information (Volkamer et al., 2001, 2002; Olariu et al., 2002).

\begin{tabular}{|c|c|c|c|c|}
\hline Hydrocarbon & $\mathrm{H}$ abstraction & & $1,2 \mathrm{OH}$ addition & \\
\hline & & Phenolic & Peroxide-bicyclic & Epoxy-oxy $^{\mathrm{a}}$ \\
\hline benzene & - & 0.53 & 0.35 & 0.12 \\
\hline toluene & 0.07 & 0.18 & 0.65 & 0.1 \\
\hline ethylbenzene ${ }^{b}$ & 0.07 & 0.18 & 0.65 & 0.1 \\
\hline o-xylene & 0.05 & 0.16 & 0.55 & 0.24 \\
\hline m-xylene & 0.04 & 0.17 & 0.5 & 0.29 \\
\hline p-xylene & 0.1 & 0.12 & 0.625 & 0.155 \\
\hline Propylbenzene ${ }^{b}$ & 0.07 & 0.18 & 0.65 & 0.1 \\
\hline i-propylbenzene ${ }^{b}$ & 0.07 & 0.18 & 0.65 & 0.1 \\
\hline 1,2,3-trimethylbenzene & 0.06 & 0.03 & 0.7 & 0.21 \\
\hline 1,2,4-trimethylbenzene & 0.06 & 0.03 & 0.61 & 0.3 \\
\hline 1,3,5-trimethylbenzene & 0.03 & 0.04 & 0.79 & 0.14 \\
\hline o-ethyltoluene ${ }^{\mathrm{c}}$ & 0.05 & 0.16 & 0.55 & 0.24 \\
\hline m-ethyltoluene ${ }^{\mathrm{c}}$ & 0.04 & 0.17 & 0.5 & 0.29 \\
\hline p-ethyltoluene ${ }^{c}$ & 0.1 & 0.12 & 0.625 & 0.155 \\
\hline 1-ethyl-3,5-dimethylbenzene ${ }^{d}$ & 0.03 & 0.04 & 0.79 & 0.14 \\
\hline 1,3-diethyl-5-methylbenzene ${ }^{\mathrm{d}}$ & 0.03 & 0.04 & 0.79 & 0.14 \\
\hline
\end{tabular}

${ }^{a}$ Epoxy-oxy route assigned balance of flux

$\mathrm{b}$ Based on data for toluene.

${ }^{c}$ Based on data for corresponding xylene

$\mathrm{d}$ Based on data for 1,3,5-trimethyl benzene

However, positive confirmation and quantification of these products has not yet been achieved. The epoxy route proceeds by formation and subsequent decomposition of a cyclic epoxy-oxy radical, and is included in MCMv3 to represent the balance of the chemistry not accounted for by other routes (Jenkin et al., 2003).

In this paper we discuss the mechanism development process which has led to an update of the aromatic mechanisms to the version MCMv3.1, and give details of the changes relative to the MCMv3 aromatic protocol (Jenkin et al., 2003). Newly available kinetic and product data from the literature have been taken into account, including results from laboratory experiments carried out as part of the EXACT project. Key subsets of the toluene system were investigated with chamber experiments and, where appropriate, the results have been used to refine the mechanisms. The lower panel of Fig. 1 shows the pathways implemented in the MCMv3.1 toluene mechanism. As discussed in detail in Sect. 2.1, the channel branching ratios have been changed, and the 1,4-addition route is no longer included as $p$-methylbenzoquinone is produced as a second generation product in the phenolic route. The oxidation mechanism of cresol has been updated and the major product of the $\mathrm{OH}$ reaction is the ring-retaining compound, methyl-catechol (3-methylbenzene-1,2-diol).
In a companion paper (Bloss et al., 2004) the performance of benzene, toluene, $p$-xylene and 1,3,5-trimethylbenzene MCMv3.1 mechanisms is evaluated using the dataset on aromatic photosmog systems, and compared to the performance of MCMv3 mechanisms. Significant discrepancies are identified in the model-measurement comparisons, and in this paper we discuss ideas for further modifications to the mechanisms to account for these shortcomings.

\section{Mechanism development using literature data}

The aromatic mechanisms, MCMv3.1, are updated versions of those included in MCMv3. The mechanism development protocol for aromatic oxidation in MCMv3 is available in the literature (Jenkin et al., 2003), and is broadly applicable to MCMv3.1. The areas where changes to the mechanism have been made based on kinetic and product data from the literature are described below. These changes apply to those compounds where $\mathrm{OH}$ attack is mainly on the aromatic ring, i.e. where substituents are saturated alkyl groups. The mechanisms for styrene and benzaldehyde, where $\mathrm{OH}$ attack is assumed to be solely on the substituent group, have not been changed. 


\subsection{Branching ratios of routes to first generation products}

The branching ratios of $\mathrm{OH}$ initiated oxidation routes to first generation products are shown in Table 1 . The previously implemented 1,4-addition route has been removed because, as described in Sect. 2.4, the appropriate $p$-benzoquinone intermediate is produced in the oxidation of the hydroxyarene (Olariu et al., 2002).

2.1.1 Benzene, toluene, $p$-xylene, $p$-ethyltoluene and other monoalkyl substituted aromatics

For benzene, toluene and $p$-xylene, branching ratios have been changed to reflect the currently available information as described below. The toluene data are also used to define the branching ratios for the other monoalkyl-substituted aromatics, and $p$-ethyltoluene is assumed to have the same branching ratios as $p$-xylene. The following changes have been made from MCMv3:

(1) H-abstraction. The benzaldehyde yield in the toluene system is slightly lower than implemented in MCMv3 to better represent the more recent data at $\mathrm{NO}_{\mathrm{x}}$ levels appropriate to polluted atmospheric conditions (Calvert et al., 2002 and references therein).

(2) Phenolic route. For benzene the phenol yield reported by Volkamer et al. (2002) is used, which is more than twice the value used in MCMv3. Volkamer et al. (2002) found this high phenol yield to be constant for $\mathrm{NO}_{\mathrm{x}}$ levels of up to around $100 \mathrm{ppb}$, i.e. appropriate levels for atmospheric conditions, but saw a decreasing yield at higher $\mathrm{NO}_{\mathrm{x}}$.

For both toluene and $p$-xylene the branching ratio of the phenolic route is decreased from that used in MCMv3. The yields of hydroxyarenes in these systems have been found to increase with increasing $\mathrm{NO}_{\mathrm{x}}$ (Atkinson and Aschmann, 1994) and the yields used in MCMv3.1 are those measured at $\mathrm{NO}_{\mathrm{x}}$ levels most appropriate for atmospheric conditions (Volkamer, 2001).

(3) Peroxide-bicyclic route. The peroxide-bicyclic route is used to describe the formation of $\alpha$-dicarbonyl products and assumed co-products, $\alpha, \beta$ unsaturated- $\gamma$-dicarbonyls and furanones. As discussed in the MCMv3 protocol (Jenkin et al., 2003) a representative alkoxy radical is used in the mechanisms and assigned several decomposition channels to account for all possible combinations of $\alpha$-dicarbonyl and co-products. In most cases the relative yields assigned to these channels is as MCMv3 but for monoalkyl-substituted aromatics the ratio of $\mathrm{HC}(\mathrm{O}) \mathrm{CHO}: \mathrm{RC}(\mathrm{O}) \mathrm{CHO}$ is changed from 1:1 to $3: 2$ to reflect the ratio of product yields measured under $\mathrm{NO}_{\mathrm{x}}$ conditions approaching those that occur in the atmosphere (Smith et al., 1998).

In the case of benzene, glyoxal is the only possible $\alpha$ dicarbonyl and the reported primary product yield of glyoxal (Volkamer et al., 2001) is used for the peroxide-bicyclic route. Volkamer et al. (2001) also measured the primary product yields of glyoxal from toluene and $p$-xylene and these are used to define the peroxide-bicyclic branching ratios for these compounds, taking into account the relative $\alpha$ dicarbonyl yields discussed above.

(4) Epoxy-oxy route. The epoxy-oxy route is assigned as the balance of the reaction flux, as in MCMv3, giving branching ratios shown in Table 1.

\subsubsection{Other aromatics}

No new product information is available for $o$ xylene, $m$-xylene, $o$-ethyltoluene, $m$-ethyltoluene, 1,2,3-trimethylbenzene, 1,2,4-trimethylbenzene, 1,3,5trimethylbenzene, 1-ethyl-3,5-dimethylbenzene, and 1,3-diethyl-5-methylbenzene. The branching ratios for $\mathrm{H}$-abstraction, phenolic and peroxide-bicyclic routes are consequently unchanged from MCMv3. The epoxy-oxy route is assigned as the balance of flux with the 1,4-addition route omitted.

\subsection{Degradation of $\alpha, \beta$ unsaturated- $\gamma$-dicarbonyls}

As discussed further below in Sect. 4.1.2, Thuener et al. (2003a) have studied the rapid photolysis of butenedial and 4-oxo-2-pentenal in the chamber at EUPHORE. They found photolysis rates of $0.14 \times \mathrm{J}_{\mathrm{NO} 2}$, and $0.2 \times \mathrm{J}_{\mathrm{NO} 2}$ for butenedial and 4-oxo-2-pentenal, respectively, and these values are used in the MCMv3.1 mechanism. A generic rate of $0.2 \times \mathrm{J}_{\mathrm{NO} 2}$ is used for all other $\alpha, \beta$ unsaturated $-\gamma$ dicarbonyls containing an aldehyde group.

The cyclic furanone compounds were found to be major products of $\alpha, \beta$ unsaturated- $\gamma$-dicarbonyl photolysis while $\alpha$-dicarbonyl products were observed only in very low yield. Therefore the following product channels are used:

$\mathrm{RC}(\mathrm{O}) \mathrm{CH}=\mathrm{CHCHO} \longrightarrow \stackrel{\mathrm{O}_{2}}{\longrightarrow} \mathrm{RC}(\mathrm{O}) \mathrm{CH}=\mathrm{CHC}(\mathrm{O}) \mathrm{O}_{2}+\mathrm{HO}_{2}(1 \mathrm{a})$

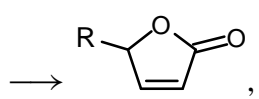

where $\mathrm{k}_{(1 \mathrm{a})} / \mathrm{k}_{(1 \mathrm{~b})}=3 / 2$. The chemistry of one such acyl peroxy radical, $\mathrm{HC}(\mathrm{O}) \mathrm{CH}=\mathrm{CHC}(\mathrm{O}) \mathrm{O}_{2}$, in the absence of $\mathrm{NO}_{\mathrm{x}}$ is illustrated in Fig. 2 and analogous schemes can be drawn for all $\mathrm{RC}(\mathrm{O}) \mathrm{CH}=\mathrm{CHC}(\mathrm{O}) \mathrm{O}_{2}$. With $\mathrm{NO}_{\mathrm{x}}$ present reactions of these acyl peroxy radicals with $\mathrm{NO}$ and $\mathrm{NO}_{2}$ become important and are implemented according to the non-aromatic MCM protocol (Jenkin et al., 1997; Saunders et al., 2003).

The MCMv3 and MCMv3.1 have been compared to chamber experiments both with and without $\mathrm{NO}_{\mathrm{x}}$, and these results are discussed in detail in Sect. 4.1. Significant discrepancies between modelled and measured concentrations were observed in all cases and it is clear that our understanding of this chemistry remains poor. Thuener et al. (2003a) propose a different photolysis mechanism for the $\gamma$-dicarbonyls in $\mathrm{NO}_{\mathrm{x}}$ free conditions involving a ketene intermediate but the major end products are similar, i.e. the appropriate cyclic 


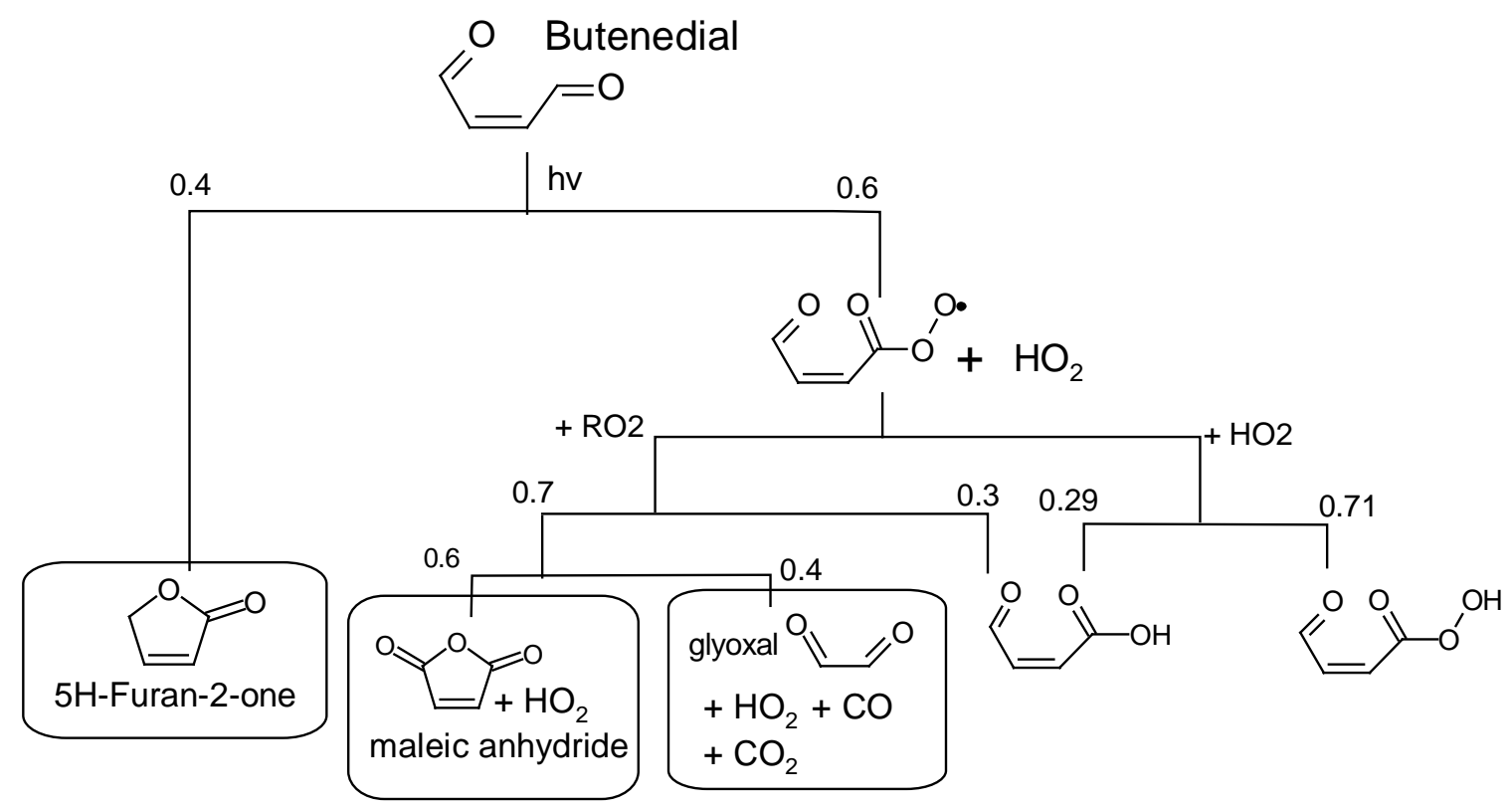

Fig. 2. Schematic representation of MCMv3.1 butenedial photolysis mechanism in the absence of $\mathrm{NO}_{\mathrm{x}}$.

furanone and maleic anhydride. Adopting this mechanism in MCM would not resolve the difficulties of simulating the chamber data and would increase complexity by introducing additional short-lived compounds into the mechanism.

For $\alpha, \beta$ unsaturated- $\gamma$-diketones the photolysis products remain unchanged from MCMv3 as product channels (1a) and (1b) involve an aldehydic $\mathrm{H}$ and are not available to the diketones. However the photolysis rates for these compounds were changed to $0.0057 \times \mathrm{J}_{\mathrm{NO} 2}$ in accordance with the measurements of Graedler and Barnes (1997).

Recent work by Bethel et al. (2001) has identified $\mathrm{CH}_{3} \mathrm{C}(\mathrm{O}) \mathrm{CH}(\mathrm{OH}) \mathrm{CHO}$ as a major product in the $\mathrm{OH}$ initiated oxidation of 3-hexene-2,5-dione and two decomposition pathways are assigned to the alkoxy radical:

$\mathrm{CH}_{3} \mathrm{C}(\mathrm{O}) \mathrm{CH}(\mathrm{OH}) \mathrm{CH}\left(\mathrm{O}^{\bullet}\right) \mathrm{C}(\mathrm{O}) \mathrm{CH}_{3}$

$\longrightarrow \stackrel{\mathrm{O}_{2}}{\longrightarrow} 2 \mathrm{CH}_{3} \mathrm{C}(\mathrm{O}) \mathrm{CHO}+\mathrm{HO}_{2}$

$\longrightarrow \stackrel{\mathrm{O}_{2}}{\longrightarrow} \mathrm{CH}_{3} \mathrm{C}(\mathrm{O}) \mathrm{CH}(\mathrm{OH}) \mathrm{CHO}+\mathrm{CH}_{3} \mathrm{C}(\mathrm{O}) \mathrm{O}_{2}$,

where $\mathrm{k}_{(2 \mathrm{a})} / \mathrm{k}_{(2 \mathrm{~b})}=3 / 7$.

Analogous reactions are implemented for other $\alpha, \beta$ unsaturated- $\gamma$-diketones.

\subsection{Degradation of furanones}

Following the observation that secondary yields of glyoxal in aromatic systems are negligible compared to the primary yields (Volkamer et al., 2001) changes were made to the MCM aromatic mechanisms to reduce the amount of glyoxal and other $\alpha$-dicarbonyl compounds formed as second generation products. A major secondary source of glyoxal in MCMv3 aromatic mechanisms was the degradation of furanone compounds.

Measured or estimated rate coefficients for $\mathrm{OH}$ and $\mathrm{NO}_{3}$ reactions were used in the standard way (Jenkin et al., 1997; Saunders et al., 2003) with addition to the double bond assumed to be the only important pathway. The difference to MCMv3 is in the assumed decomposition of the resultant alkoxy radicals; in MCMv3.1 the $\mathrm{C}(\mathrm{O} \bullet)-\mathrm{C}(\mathrm{OH})$ or $\mathrm{C}(\mathrm{O} \bullet)$ $\mathrm{C}\left(\mathrm{NO}_{3}\right)$ breaks and the product is a compound with an ester linkage, e.g.:<smiles>CC(=O)C(=O)OCC=O</smiles>

The further degradation of this product follows the nonaromatic MCM protocol and in most cases $\alpha$-dicarbonyl compounds are not major products of this chemistry.

The $\mathrm{O}_{3}$ initiated degradation of furanones is included with updated rate coefficients and products. The reaction of $2(5 \mathrm{H})$-furanone with $\mathrm{O}_{3}$ has been studied by Grosjean and Grosjean (1999) and the measured rate coefficient, $2.2 \times 10^{-19} \mathrm{~cm}^{3}$ molecule ${ }^{-1} \mathrm{~s}^{-1}$ is used in MCMv3.1. The alkyl-substituted furanones with structures $(-\mathrm{CH}=\mathrm{CR}-)$ and $(-\mathrm{CR}=\mathrm{CR}-)$ are assigned generic rate coefficients of $8 \times 10^{-19} \mathrm{~cm}^{3}$ molecule $\mathrm{s}^{-1}$ and $1.4 \times 10^{-18} \mathrm{~cm}^{3}$ molecule $\mathrm{s}^{-1} \mathrm{~s}^{-1}$, respectively, to reflect the greater rate expected with greater substitution of the double bond. In most cases the excited Criegee biradical formed in the ozonolysis reaction is assumed to solely decompose 
to generate $\mathrm{OH}$ radicals. For $2(5 \mathrm{H})$-furanone and angelicalactone the stabilisation pathway is also included with a stabilisation to decomposition ratio of 1:1.

In addition the representation of angelica-lactone has been changed to $\alpha$-angelica-lactone from $\beta$-angelica-lactone as the $\alpha$ form is that seen experimentally (Smith et al., 1998).

\subsection{Degradation of hydroxyarenes}

The chemistry of the hydroxyarenes was updated following new product studies indicating far higher yields of ringretaining products in these systems than previously assumed (Olariu, 2001; Olariu et al., 2002). Further studies have also been carried out on the reaction kinetics of these products (Olariu et al., 2000; Rea et al., 2001) and all the available information was used to update the mechanisms in MCMv3.1. The photo-oxidation chemistry of $o$-cresol was investigated in EUPHORE, and as discussed below in Sect. 4.2 the results of this experiment were used to refine the toluene mechanism, and by analogy other aromatic mechanisms.

However, in the case of 1,3,5-alkyl benzenes, the arrangement of the alkyl substituents precludes the formation of 1,2dihydroxyarenes and $p$-benzoquinone products analogous to those observed in other aromatic systems. Therefore the degradation mechanisms for hydroxyarenes formed by oxidation of 1,3,5-alkyl benzenes are unchanged from MCMv3. As shown in Table 1, the phenolic yield for these aromatics is very low and this channel does not have a large influence on the chemistry as a whole.

\subsubsection{Branching ratios for $\mathrm{OH}$ initiated degradation}

Three initial channels are implemented for $\mathrm{OH}$ reaction with hydroxyarenes:

1. Formation of a 1,2-dihydroxyarene product and $\mathrm{HO}_{2}$ probably by sequential 1,2 addition of $\mathrm{OH}$ and $\mathrm{O}_{2}$ to the aromatic ring followed by isomerisation and decomposition.

2. Formation of a phenoxy-type radical, which is assumed to react with $\mathrm{NO}_{2}$ to generate a hydroxy-nitroarene.

3. Formation of a bicyclic peroxy radical similar to that described above for the parent aromatic. The fate of the alkoxy radical is split between two pathways; a ring-opening channel forming an $\alpha$-dicarbonyl and corresponding hydroxy-substituted $\alpha, \beta$-unsaturated- $\gamma$ dicarbonyl, and a rearrangement to a $p$-benzoquinone product.

The yields of each channel in each aromatic degradation mechanism are shown in Table 2. As discussed above, for the 1,3,5-alkyl substituted aromatic hydrocarbons these are unchanged from MCMv3.

As described in the aromatic MCMv3 protocol (Jenkin et al., 2003), a single representative hydroxyarene product is generated from each parent aromatic hydrocarbon. The subsequent chemistry of this product is constructed to take into account the reactions of all possible isomers. For example in the toluene mechanism $o$-cresol is used to represent the three possible isomers. Product studies have been carried out for all three isomers (Olariu et al., 2002) and weighted averages of the channel yields of cresol oxidation are assigned to $o$-cresol in the MCMv3.1 mechanism based on the relative yields of $o$-cresol, $m$-cresol and $p$-cresol from toluene oxidation (Calvert et al., 2002 and references therein).

Product yields of 1,2-dihydroxyarenes and hydroxynitroarenes are available for phenol and the cresol isomers (Olariu et al., 2002). In general the values for cresol are also used for the other alkyl-substituted hydroxyarenes. However, in some cases it is not possible to form a 1,2-dihydroxyarene from one or more of the possible hydroxyarene isomers and the 1,2-dihydroxyarene yield is reduced taking into account the relative yields of each isomer from the parent aromatic oxidation.

Benzoquinone yields are available for phenol, all cresol isomers, 2,3-dimethylphenol and 2,6-dimethylphenol, (Rea et al., 2001; Olariu et al., 2002), and these define the yields used in the benzene, toluene, $m$-xylene and $o$-xylene systems. The other mono- and di-substituted aromatic hydrocarbon systems have yields assigned by analogy. Among the considered trimethylbenzenes only the 1,2,4-isomer can form p-benzoquinone via two of its three hydroxyarenes (which account for $77 \%$ of the total hydroxyarenes here). A $20 \%$ yield was assumed to derive a p-benzoquinone yield from 1,2,4-trimethylbenzene of $15 \%$.

In all cases the balance of the reaction is assumed to give ring-opened products, i.e. $\alpha$-dicarbonyls and the corresponding hydroxy-substituted $\alpha, \beta$-unsaturated- $\gamma$-dicarbonyls.

\subsubsection{Branching ratios for $\mathrm{NO}_{3}$ initiated degradation}

Three initial channels are implemented for $\mathrm{NO}_{3}$ reaction with hydroxyarenes to account for the observed products:

1. Formation of a phenoxy-type radical and $\mathrm{HNO}_{3}$. The phenoxy radical is as channel 2 of the $\mathrm{OH}$ initiated chemistry, and assumed to react with $\mathrm{NO}_{2}$ to generate a hydroxy-nitroarene.

2. Formation of $\mathrm{HNO}_{3}$ and the bicyclic peroxy radical described above for channel 3 of the $\mathrm{OH}$ initiated chemistry. The products of this channel are then $\alpha$ dicarbonyls and corresponding hydroxy-substituted $\alpha$, $\beta$-unsaturated- $\gamma$-dicarbonyls, and $p$-benzoquinones.

3. Addition of $\mathrm{NO}_{3}$ to the ring and formation of a nitrooxy-substituted bicyclic peroxy radical. The subsequent chemistry leads to an alkoxy radical that decomposes to give $\mathrm{NO}_{2}$ and the same $\alpha$-dicarbonyls and corresponding hydroxy-substituted $\alpha, \beta$-unsaturated- $\gamma$ dicarbonyls as channel 2. No $p$-benzoquinone is formed in this channel. 
Table 2. Relative branching ratios assigned to $\mathrm{OH}$ initiated oxidation pathways of first generation phenolic products in MCMv3.1, assigned on the basis of product studies (Rea et al., 2001; Olariu et al., 2002) as detailed in the text.

\begin{tabular}{|c|c|c|c|c|}
\hline \multirow[t]{2}{*}{ Hydrocarbon } & \multirow{2}{*}{$\begin{array}{c}1,2- \\
\text { dihydroxyarene }^{\text {a }}\end{array}$} & \multirow[t]{2}{*}{ Phenoxy } & \multicolumn{2}{|c|}{ Peroxide-bicyclic } \\
\hline & & & Ring-open ${ }^{b}$ & p-benzoquinone ${ }^{c}$ \\
\hline benzene & 0.8 & 0.06 & 0.1 & 0.04 \\
\hline toluene & 0.73 & 0.07 & 0.14 & 0.06 \\
\hline ethylbenzene & 0.73 & 0.07 & 0.14 & 0.06 \\
\hline o-xylene & 0.73 & 0.07 & 0.1 & 0.1 \\
\hline m-xylene & 0.42 & 0.07 & 0.43 & 0.08 \\
\hline p-xylene & 0.73 & 0.07 & 0.14 & 0.06 \\
\hline propylbenzene & 0.73 & 0.07 & 0.14 & 0.06 \\
\hline i-propylbenzene & 0.73 & 0.07 & 0.14 & 0.06 \\
\hline 1,2,3-trimethylbenzene & 0.73 & 0.07 & 0.2 & 0 \\
\hline 1,2,4-trimethylbenzene & 0.26 & 0.07 & 0.52 & 0.15 \\
\hline 1,3,5-trimethylbenzene ${ }^{d}$ & 0 & 0.05 & 0.95 & 0 \\
\hline o-ethyltoluene & 0.73 & 0.07 & 0.1 & 0.1 \\
\hline m-ethyltoluene & 0.42 & 0.07 & 0.43 & 0.08 \\
\hline p-ethyltoluene & 0.73 & 0.07 & 0.14 & 0.06 \\
\hline 1-ethyl-3,5-dimethylbenzene ${ }^{\mathrm{d}}$ & 0 & 0.05 & 0.95 & 0 \\
\hline 1,3-diethyl-5-methylbenzene ${ }^{\mathrm{d}}$ & 0 & 0.05 & 0.95 & 0 \\
\hline
\end{tabular}

${ }^{a}$ Branching ratios in benzene and toluene systems based on measured yields, others as for toluene but adjusted to account for hyroxyarene isomers where 1,2-dihydroxyarene formation is not possible.

$\mathrm{b}$ Ring-open route assigned balance of flux.

${ }^{c}$ Branching ratios in benzene, toluene, m-xylene and o-xylene systems based on measured yields, others assigned by analogy but adjusted to account for hyroxyarene isomers where p-benzoquinone formation is not possible.

$\mathrm{d}$ Branching ratios as in MCMv3 as formation of 1,2-dihydroxyarene and p-benzoquinone prevented by arrangement of alkyl substituents.

The measured yields of hydroxy-nitroarenes from $\mathrm{NO}_{3}$ initiated oxidation of phenol and cresol are used to determine the branching ratios for channel 1 of $74 \%$ and $39 \%$ in the benzene and toluene systems, respectively (Olariu, 2001).

In the case of phenol oxidation by $\mathrm{NO}_{3} p$-benzoquinone product has not been observed. Therefore the yield of channel 2 in the benzene system is set to zero and the balance of the reaction goes via channel 3 .

Small $p$-benzoquinone yields have been observed from the $\mathrm{NO}_{3}$ initiated oxidation of $o-$ and $m$-cresol (Olariu, 2001) and this information was used to assign a yield of $10 \%$ to channel 2 in the toluene system. As for benzene, channel 3 is used to account for the balance of the reaction.

In the absence of any information on other hydroxyarenes the branching ratios for cresol oxidation are applied.

\subsubsection{Degradation mechanisms of intermediates}

The ring-opened products and $p$-benzoquinones are further degraded according to the standard non-aromatic MCM protocol (Jenkin et al., 1997; Saunders et al., 2003). The other main products of hydroxyarene degradation are 1,2dihydroxyarenes and hydroxy-nitroarenes. Product studies of the oxidation of these compounds are not available and a provisional representation of their degradation was developed by analogy to the observed behaviour of the cresol isomers. However, when compared to the chamber experiment discussed below in Sect. 4.2, this mechanism leads to excess production of ozone. Adjustments to the mechanism were made and are discussed further in Sect. 4.2 and shown in Fig. 3.

\section{Mechanism testing and further developments: meth- ods}

\subsection{Identification of key intermediates}

The approach taken to test the details of the toluene oxidation mechanism was to identify key intermediates in the chemical system and perform separate experiments on those compounds. Comparison of modelled and measured concentrations in these photosmog experiments tests our understanding of these important sections of the chemistry.

The sensitivity of $\mathrm{OH}$ and $\mathrm{O}_{3}$ concentrations in the toluene system to concentrations of intermediates was investigated by Wagner et al. (2003). The highest $\mathrm{O}_{3}$ and $\mathrm{OH}$ sensitivities in VOC limited conditions were to changes in the concentration of 2,3-epoxy-6-oxo-4-heptenal and conjugated 


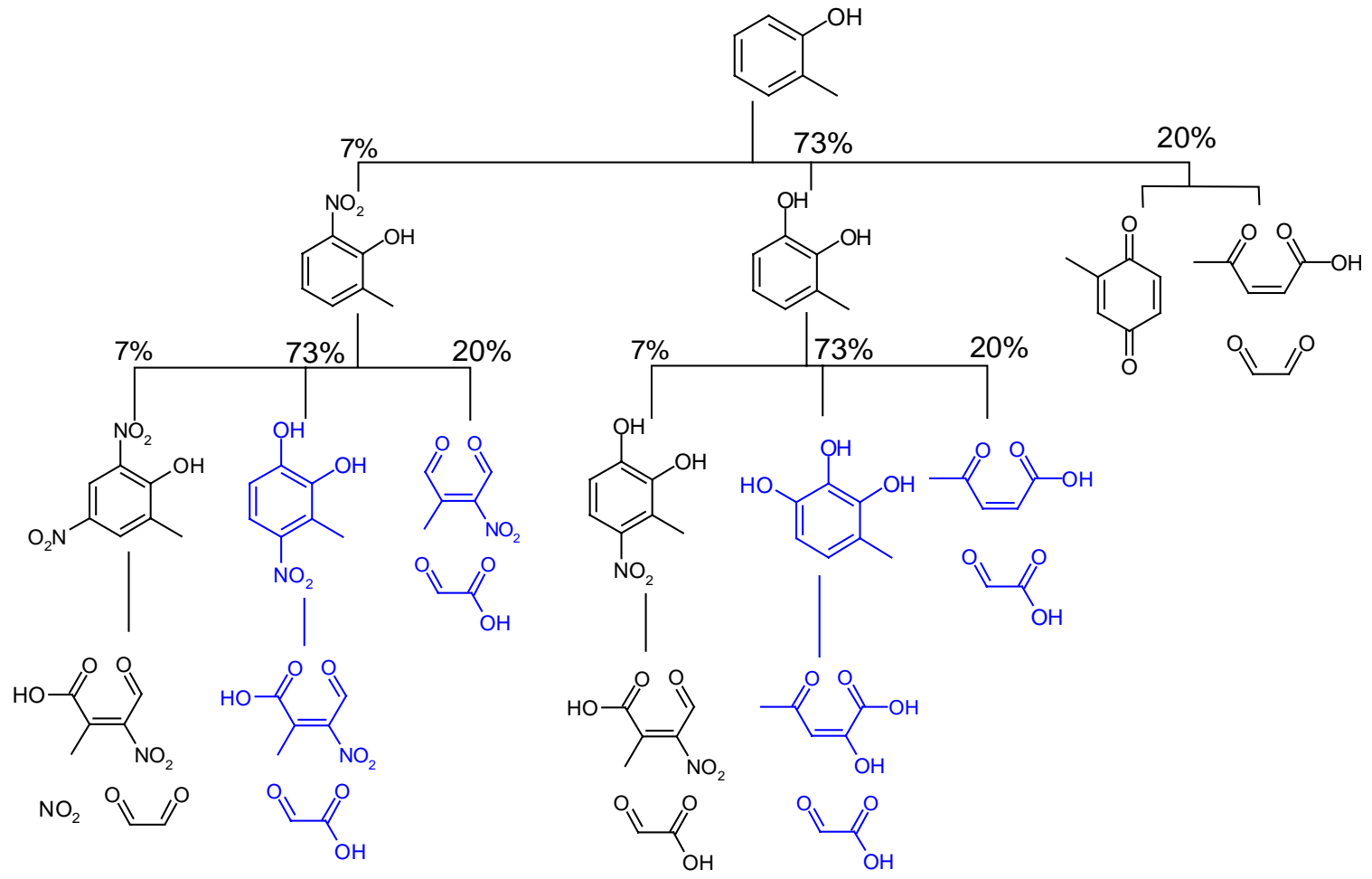

Fig. 3. Schematic representation of $\mathrm{OH}$ initiated oxidation of cresol in MCMv3.1 and MCMv3.1a; pathways shown in blue are active only in MCMv3.1a.

$\gamma$-dicarbonyls. Based on considerations of synthetic feasibility the two $\gamma$-dicarbonyls, butenedial and 4-oxo-2-pentenal were chosen for study in EUPHORE chamber experiments. The sensitivity analysis of Wagner et al. (2003) was also used to identify target compounds for GC studies aiming to improve the understanding of the carbon balance in the toluene system. These studies are discussed below and by Hamilton et al. (2003).

The ozone and $\mathrm{OH}$ in the toluene system were less sensitive to changes in the cresol concentration. However, as discussed above in Sect. 2.4, a laboratory study finding high yields of ring-retaining products from cresol oxidation suggested that large changes were needed to the representation of this chemistry in MCMv3 (Olariu et al., 2002). Therefore, it was decided to carry out a chamber photosmog experiment in order to test our understanding of the chemistry in this route.

\subsection{Experimental design}

The experiments discussed in this paper were carried out at EUPHORE in the same way as the aromatic photosmog experiments described in the companion paper (Bloss et al., 2004). The $\gamma$-dicarbonyl compounds were synthesised as described by Hamilton et al. (2003), and known quantities determined by weight were introduced into the chamber either in a heated gas stream or dissolved in $0.5-1 \mathrm{ml}$ of HPLC grade acetonitrile sprayed into the chamber in a high pressure stream of purified air. The parent hydrocarbon concentrations were measured by FTIR and for cresol an additional measurement by HPLC was available. A comprehensive gas chromatography $(\mathrm{GC} \times \mathrm{GC})$ technique was employed to identify and quantify unsaturated carbonyl intermediates (Hamilton et al., 2003). Measurements of $\mathrm{HO}_{\mathrm{x}}$ radical concentrations were made using the FAGE (Fluorescent Assay by Gas Expansion) technique; air is sampled by gas expansion and $\mathrm{OH}$ concentrations are measured by LIF (Laser Induced Fluorescence). $\mathrm{HO}_{2}$ concentrations can be measured by using $\mathrm{NO}$ to convert $\mathrm{HO}_{2}$ to $\mathrm{OH}$ before the LIF measurement. $\mathrm{OH}$ concentrations were measured during the cresol and $\gamma$ dicarbonyl photosmog experiments, and for one butenedial photosmog experiment, (4 July 2002), $\mathrm{HO}_{2}$ measurements were also made. Measurements of $\mathrm{HO}_{2}$ concentration during $\mathrm{NO}_{\mathrm{x}}$ free photolysis experiments on $\gamma$-dicarbonyls provide important information on the radical yield of these processes.

Initial conditions for the chamber experiments were carefully chosen with the aid of models, and the concentrations employed are listed in Table 3. The aim of the cresol and $\gamma$-dicarbonyl photosmog experiments was to test the model performance particularly concerning ozone production. The mechanistic details in these areas are uncertain and initial $\mathrm{VOC} / \mathrm{NO}_{\mathrm{x}}$ conditions were chosen such that ozone formation is predicted to be near maximum, thus ensuring the 
Table 3. Initial concentrations employed in simulations of chamber experiments, concentrations measured before opening chamber to sunlight.

\begin{tabular}{llllllllllll}
\hline \multirow{2}{*}{ Date } & \multirow{2}{*}{ Parent VOC } & \multicolumn{1}{c}{ VOC } & $\mathrm{NO}$ & $\mathrm{NO}_{2}$ & $\mathrm{HONO}$ & $\mathrm{O}_{3}$ & $\mathrm{HCHO}$ & $\mathrm{HNO}_{3}$ & glyoxal & $\mathrm{CO}$ & Notes \\
\hline $02 / 10 / 01$ & Butenedial & 641 & 82.3 & 36.4 & $0.1^{a}$ & 0.7 & 0.4 & - & 0.4 & 424 & Photosmog \\
$04 / 07 / 02$ & Butenedial & 243 & 122 & 0.9 & 1.1 & 0 & 0.1 & 0.6 & 0 & 610 & experiments \\
$03 / 10 / 01$ & 4-oxopentenal & 303 & 162 & 23 & 1.5 & 0 & 0 & 1.2 & 0.5 & 390 & on subsets of \\
$04 / 10 / 01$ & Cresol & 297 & 23.5 & 22.6 & 65 & 0.1 & 0.7 & 0.7 & 0 & 384 & toluene mechanism \\
\hline $05 / 07 / 02$ & Toluene & 6049 & 413 & 3.2 & 0.9 & 0 & 0.3 & 0.5 & 1.0 & 646 & Experiments to \\
$10 / 07 / 02$ & Toluene & 4737 & 464 & 0.1 & 0.1 & 0.7 & 0 & 0 & 0 & 629 & maximise concentrations \\
$12 / 07 / 02$ & Toluene & 11233 & 501 & 2.1 & 0.2 & 0 & 0.4 & 0.1 & 0 & 556 & of intermediates \\
\hline
\end{tabular}

attainment of sufficiently large ozone concentrations to be reliably measured in the chamber. An additional butenedial photosmog experiment was included to explore the effects of changing $\mathrm{VOC} / \mathrm{NO}_{\mathrm{x}}$ ratio on the measurements and the simulations.

A number of experiments were also carried out to investigate the photolysis of butenedial and 4-oxo-2-pentenal in the absence of $\mathrm{NO}_{\mathrm{x}}$. The primary aims of these experiments were to determine photolysis rates and product yields, including $\mathrm{HO}_{2}$ radical yield, and full details will be given in a forthcoming publication by Thuener et al. (in preparation, $2005)^{1}$. However, these experimental results could also be used to test the performance of $\mathrm{MCM}$ in $\mathrm{NO}_{\mathrm{x}}$ free conditions.

The aim of the set of toluene photo-oxidation experiments was to maximise the concentrations of the co-products of $\alpha$-dicarbonyls to better determine their identities and yields. We employed a strategy of using high initial concentrations of toluene and $\mathrm{NO}_{\mathrm{x}}$ while closing the chamber at the point in the experiment where maximum concentrations of butenedial and 4-oxo-2-pentenal are expected from the model simulations. In this way, loss by photolysis and $\mathrm{OH}$ reaction are prevented and the concentrations are kept high for detection by analytical methods. The choice of initial toluene concentrations was constrained by the occurrence of interference with $\mathrm{GC} \times \mathrm{GC}$ measurements at high toluene concentrations, and the configuration of the NO monitor meant that initial NO concentrations were limited to a maximum of $500 \mathrm{ppb}$. In any case it is desirable to keep NO concentrations below around $500 \mathrm{ppb}$ as at higher concentrations reaction of the 1,2-hydroxycyclohexadienyl radical with NO becomes competitive with the unimolecular decomposition and rearrangement reactions (Klotz et al., 2002). This competition is not representative of the situation in ambient conditions and is therefore not represented in MCM.

\footnotetext{
${ }^{1}$ Thuener, L. P., Rea, G., Wenger, J., Henderson, A. P., Bleasdale, C., and Golding, B. T.: Photolysis of Butenedial and 4oxopent-2-enal under atmospheric conditions, Environ. Sci. Technol., in preparation, 2005.
}

\subsection{Model simulations of smog-chamber experiments}

The simulations were initialised with measured concentrations as listed in Table 3. Where measurements of HONO were not available ( 2 and 3 October 2001), the initial HONO concentrations were estimated from chamber characterisation experiments as described in the companion paper (Bloss et al., 2005). However, for these experiments with photolabile $\gamma$-dicarbonyl compounds, HONO photolysis is not a significant radical source and the choice of initial concentration within the range $0.1-1.5 \mathrm{ppb}$ has a negligible effect on the simulations.

The temperature and humidity of the chamber were continuously monitored during the experiments and these values are used in the models. The measured photolysis rate for $\mathrm{NO}_{2}$ is available and for all other photolysis processes calculated rates were used with scaling factors applied to account for the transmission of the chamber walls, backscattering from the chamber floor and cloud cover. The scaling factors are discussed in more detail in the companion paper (Bloss et al., 2005).

An auxiliary mechanism was employed to take into account chamber related effects. The development of this mechanism is described in the companion paper and the same reactions are used in the simulations discussed below.

\section{Mechanism testing and further developments: results}

\subsection{Photo-oxidation of $\gamma$-dicarbonyls}

\subsubsection{Photo-smog systems: in the presence of $\mathrm{NO}_{\mathrm{x}}$}

Three experiments were carried out to investigate the photooxidation of $\gamma$-dicarbonyls in the presence of $\mathrm{NO}_{\mathrm{x}}$, two with butenedial at different $\mathrm{VOC} / \mathrm{NO}_{\mathrm{x}}$ ratios and one with 4 oxo-2-pentenal in similar conditions to the lower $\mathrm{VOC} / \mathrm{NO}_{\mathrm{x}}$ butenedial experiment. The initial conditions for all these experiments are given in Table 3.

As outlined above in Sect. 2.2, the mechanisms and rates of $\gamma$-dicarbonyl photolysis have been updated in MCMv3.1. 

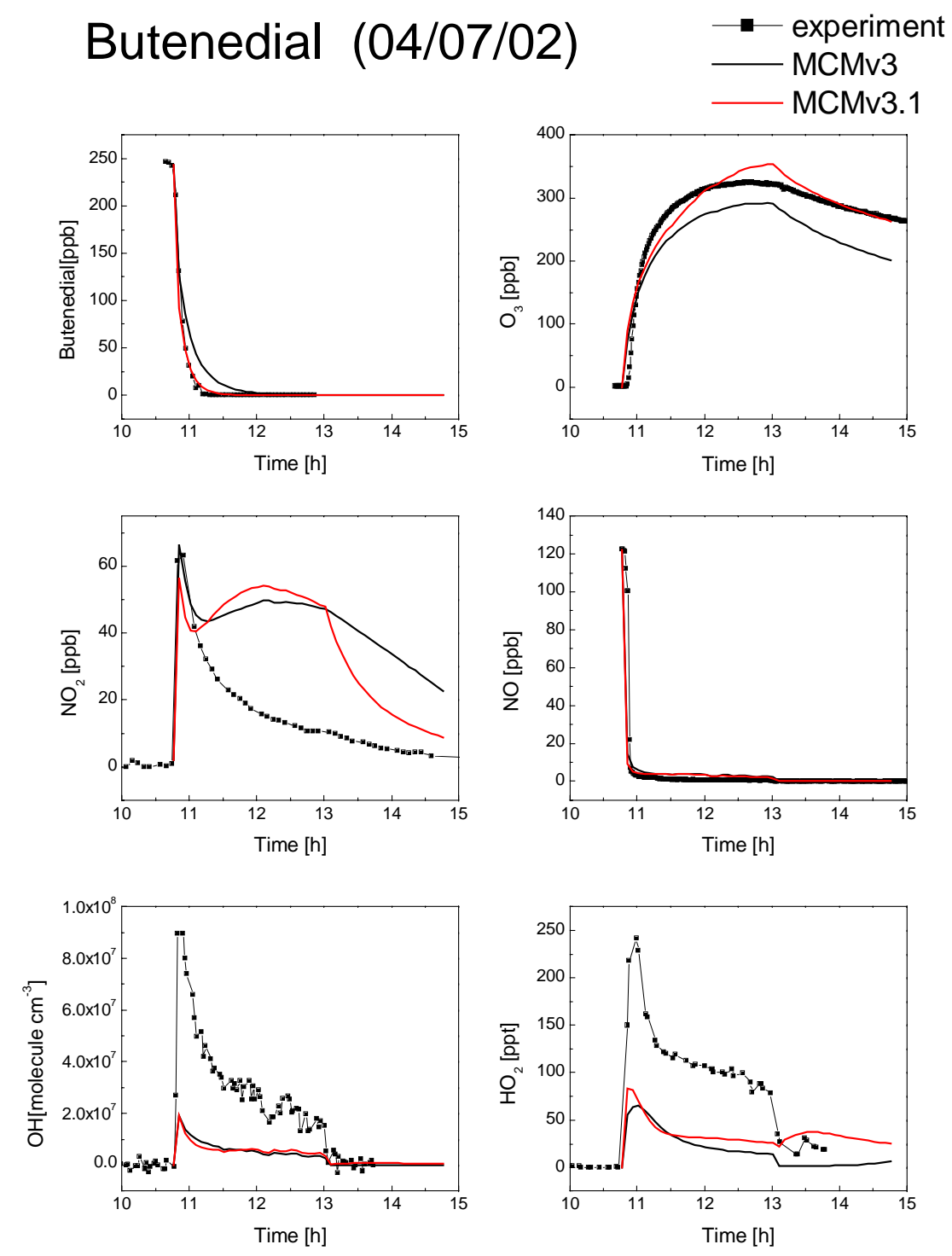

Fig. 4. Butenedial photosmog experiment (4 July 2002) where the chamber was closed at 13:00 h; comparison of modelled and measured concentrations of parent compound, $\mathrm{O}_{3}, \mathrm{NO}_{\mathrm{x}}$ and $\mathrm{HO}_{\mathrm{x}}$.

The $\mathrm{OH}$ initiated chemistry for these two $\gamma$-dicarbonyls has not been changed from MCMv3.

Comparisons of model simulations using MCMv3 and MCMv3.1 for the lower $\mathrm{VOC} / \mathrm{NO}_{\mathrm{x}}$ butenedial experiment (4 July 2002) are shown in Figs. 4 and 5. The loss of the parent compound, the $\mathrm{O}_{3}$ formation, $\mathrm{NO}_{\mathrm{x}}$ concentrations and $\mathrm{HO}_{\mathrm{x}}$ radical concentrations are given in Fig. 4, and Fig. 5 shows the simulated and measured concentrations of some product species.

The removal of butenedial is well simulated by MCMv3.1 with a faster removal than simulated by MCMv3 because of the increased photolysis rate coefficient. However butenedial is also removed by reaction with $\mathrm{OH}$ and the simulated $\mathrm{OH}$ concentration is much lower than measured. An increase of $\mathrm{OH}$ concentration in the model would lead to an over-estimate of the butenedial removal rate with the current photolysis and reaction rates implemented. If the photolysis rate measured in the EUPHORE chamber (Thuener et al., 2003a) is assumed to be correct, the rate coefficient for $\mathrm{OH}$ reaction would need to be reduced by about a factor of 3 to give model-measurement agreement for butenedial loss with the measured $\mathrm{OH}$ concentrations. The rate coefficient implemented is the only literature value available, $5.21 \times 10^{-11} \mathrm{~cm}^{3}$ molecule ${ }^{-1} \mathrm{~s}^{-1}$ (Bierbach et al., 1994), and is for cis-butenedial, whereas in the chamber experiment trans-butenedial was used. Bierbach et al. (1994) also studied trans-butenedial+OH but, because of trans/cis photo-isomerisation in their system, were 


\section{Butenedial (04/07/02)}
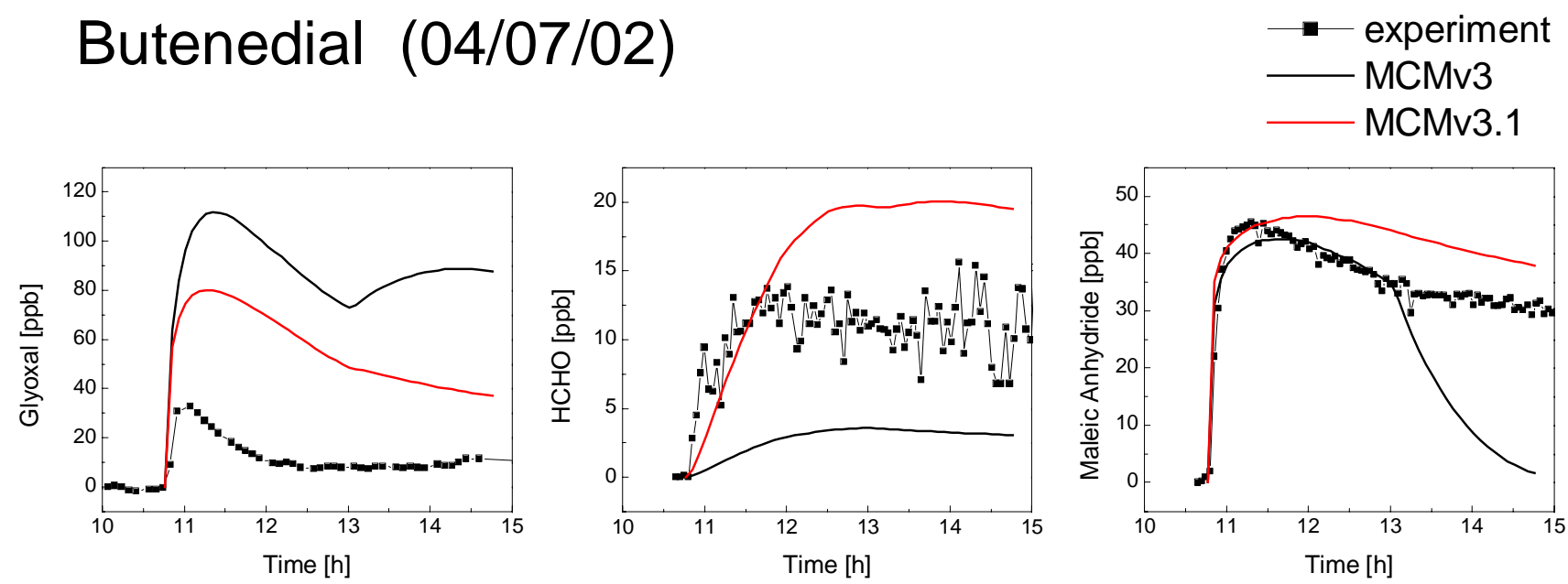

Fig. 5. Butenedial photosmog experiment (4 July 2002) where the chamber was closed at 13:00 h; comparison of modelled and measured concentrations of key intermediates.

only able to give a lower limit for this rate coefficient, $(2.41 \pm 0.79) \times 10^{-11} \mathrm{~cm}^{3}$ molecule $\mathrm{e}^{-1} \mathrm{~s}^{-1}$. Therefore it is possible that for simulation of this chamber experiment a lower rate coefficient for $\mathrm{OH}$ reaction may be appropriate. Using a rate coefficient of $1.8 \times 10^{-11} \mathrm{~cm}^{3}$ molecule ${ }^{-1} \mathrm{~s}^{-1}$ for this reaction results in an increased $\mathrm{OH}$ concentration in the first $15 \mathrm{~min}$ of the experiment, but still underestimates the observed concentration at this time by more than a factor of 10 , and has no significant effect on the overall simulation. The MCM should be appropriate for atmospheric conditions, and, as it is not clear which isomer dominates in atmospheric conditions, in the absence of further information the literature value for cis-butenedial is used in MCMv3.1 as in MCMv3.

The behaviour of the two simulations is broadly similar while the chamber is open and photolysis pathways and $\mathrm{OH}$ reactions are active. The differences that are evident after the chamber closes at 13:00 $\mathrm{h}$ are a result of changes to the $\mathrm{NO}_{3}$ chemistry and are discussed further below. However, there are large discrepancies between the simulations and the measured concentrations throughout the experiment. The increased photolysis rate and altered product distribution of MCMv3.1 results in a small increase in the simulated $\mathrm{HO}_{2}$ concentrations, but the concentrations of $\mathrm{OH}$ and $\mathrm{HO}_{2}$ radicals are under-predicted by more than a factor of 3 throughout the experiment. The $\mathrm{NO}_{2}$ concentration profiles clearly demonstrate that the $\mathrm{NO}_{\mathrm{x}} / \mathrm{NO}_{\mathrm{y}}$ chemistry is poorly understood. The initial loss of $\mathrm{NO}$ and production of $\mathrm{NO}_{2}$ is well simulated but after 11:00 $\mathrm{h}$ the simulations show a secondary increase in $\mathrm{NO}_{2}$ concentration which is not seen experimentally. This is a result of the formation and subsequent decomposition of PAN type compounds when acyl peroxy radicals react with $\mathrm{NO}_{2}$. Given these large discrepancies, the ozone concentration is surprisingly well predicted; the peak ozone is over-predicted by $9 \%$ by MCMv3.1 and under-predicted by $10 \%$ by MCMv3. This good agreement is perhaps coincidental, and, as discussed below, is not seen in the other $\gamma$-dicarbonyl experiments. The under-prediction of radical concentrations in general reduces the ozone production while an over-prediction of $\mathrm{NO}_{2}$ increases ozone concentration as $\mathrm{NO}_{2}$ photolysis produces ozone.

The concentration time profiles for some important intermediates are shown in Fig. 5. The glyoxal concentration is considerably lower in the MCMv3.1 simulations as it is no longer directly produced by photolysis. However, the simulated concentration is still significantly higher than that measured. For formaldehyde MCMv3 significantly underpredicts the concentration. HCHO production is increased by the changed product distribution in MCMv3.1 and in this case the measured concentration is over-predicted. The yield of maleic anhydride is similar in the two simulations and the peak concentration predicted by MCMv3.1 is in excellent agreement with the measurement. MCMv3 predicts a rapid decrease in maleic anhydride concentration when the chamber closes, and this is not seen experimentally. The reaction of maleic anhydride with $\mathrm{NO}_{3}$ is assigned a relatively high rate coefficient in MCMv3 and it is this process that is responsible for the efficient removal of maleic anhydride. The $\mathrm{NO}_{3}$ concentration in the model is unrealistically high because of the large over-prediction of $\mathrm{NO}_{2}$; in the dark the reaction of $\mathrm{NO}_{2}$ with $\mathrm{O}_{3}$ leads to a build up of $\mathrm{NO}_{3}$. However, even if $\mathrm{NO}_{2}$ is constrained to the measurement reducing $\mathrm{NO}_{3}$ to a more reasonable level, the simulated loss rate for maleic anhydride is much higher than observed. The simulated increase of glyoxal concentration in the dark occurs because glyoxal is a product of this $\mathrm{NO}_{3}$ initiated chemistry, and this is also not observed in the experimental data. There are no literature values for this rate 


\section{- 02/10/01 - 4-oxo-2-pentenal $\left(\mathrm{VOC} / \mathrm{NO}_{\mathrm{x}}=1.6\right)$ \\ $-03 / 10 / 01$ - butenedial $\left(\mathrm{VOC} / \mathrm{NO}_{\mathrm{x}}=5.4\right)$}
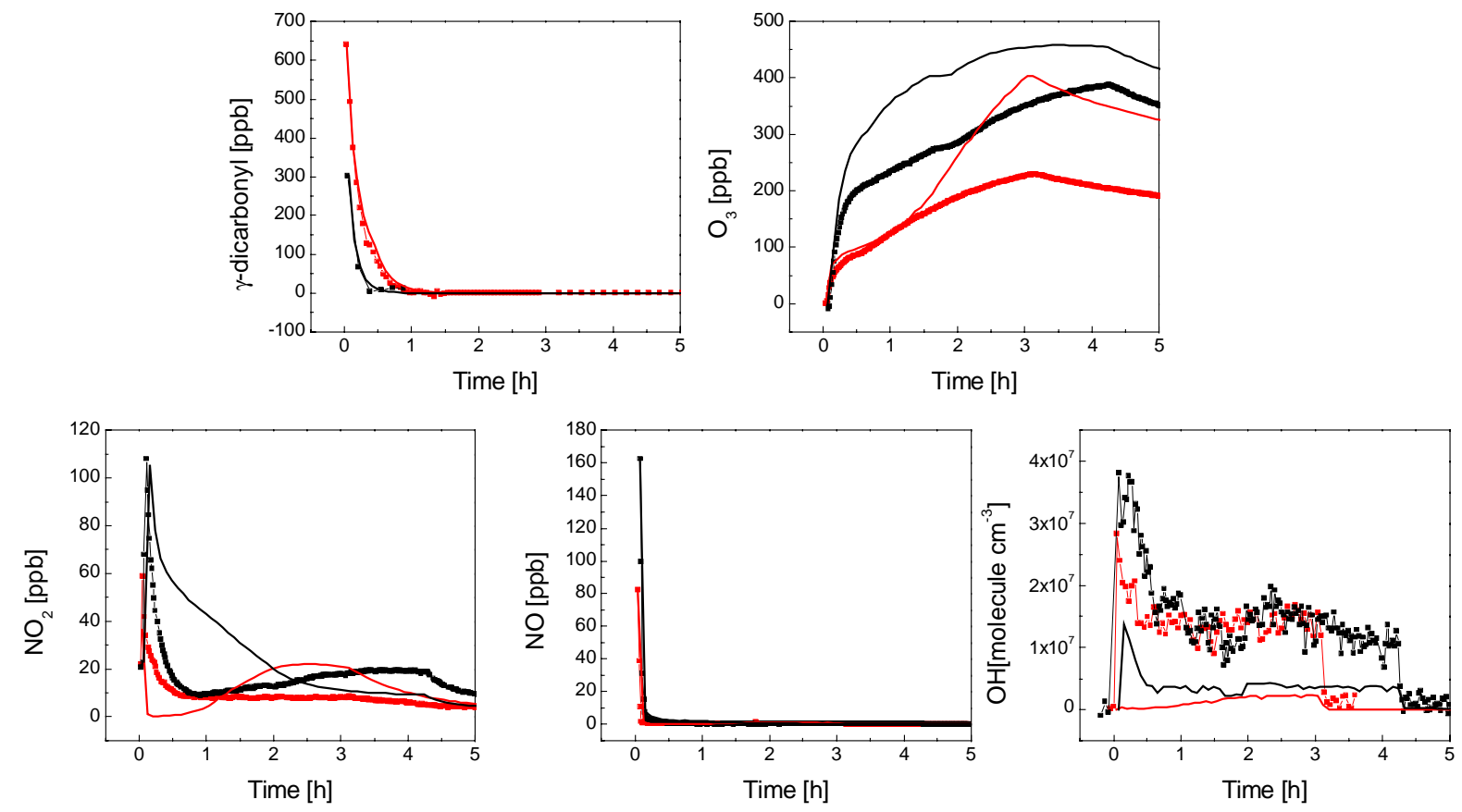

Fig. 6. $\gamma$-Dicarbonyl photosmog experiments (2 and 3 October 2001); comparison of modelled (MCMv3.1) and measured concentrations of parent compound, $\mathrm{O}_{3}, \mathrm{NO}_{\mathrm{x}}$ and $\mathrm{OH}$.

coefficient but Bierbach et al. (1994) estimated it to be less than $10^{-18} \mathrm{~cm}^{3}$ molecule $\mathrm{e}^{-1} \mathrm{~s}^{-1}$ based on structure-reactivity comparisons. As no evidence for the reaction was seen in the chamber experiment, and it is expected to be slow; it is not included in MCMv3.1. This reaction was an important sink for $\mathrm{NO}_{3}$ in $\mathrm{MCMv} 3$ and consequently the $\mathrm{NO}_{3}$ concentrations in MCMv3.1 after the chamber closes are much higher. This high $\mathrm{NO}_{3}$ concentration results in the increased $\mathrm{NO}_{2}$ loss rate and also the production of $\mathrm{HO}_{2}$ in the dark by reaction of $\mathrm{NO}_{3}$ with glyoxal and formaldehyde.

The MCMv3.1 simulations of the parent compound, $\mathrm{O}_{3}$, $\mathrm{OH}$ and $\mathrm{NO}_{\mathrm{x}}$ concentrations for the other two $\gamma$-dicarbonyl experiments (2 and 3 October 2001) are compared to the measurements in Fig. 6. The time axis has been shifted to aid comparison between experiments and zero is the start time of the experiment, when the chamber was opened. As discussed above, the $\gamma$-carbonyl loss rates are well simulated by MCMv3.1 despite the large under-prediction of $\mathrm{OH}$ concentrations. The measured $\mathrm{OH}$ concentrations are very similar in the two experiments showing a peak in the first half hour of the experiment and remaining at a fairly steady level of about $1.5 \times 10^{7}$ molecule $\mathrm{cm}^{-3}$ in the later stages. The peak concentrations are lower than observed in the lower $\mathrm{VOC} / \mathrm{NO}_{\mathrm{x}}$ butenedial experiment (4 July 2002) and in that experiment the $\mathrm{OH}$ concentration showed a steady decline from the peak rather than levelling off. The simulated $\mathrm{OH}$ in the 4-oxo2-pentenal experiments follows a similar time profile to the observations albeit at a lower concentration by about a factor of 3. In the high $\mathrm{VOC} / \mathrm{NO}_{\mathrm{x}}$ butenedial case no early $\mathrm{OH}$ peak is predicted and the simulation shows a slow increase in $\mathrm{OH}$ concentration through the experiment, but even at the end of the experiment it under-predicts the measurement by about a factor of 6 .

The measured ozone concentration time profiles for the 4oxo-2-pentenal and high $\mathrm{VOC} / \mathrm{NO}_{\mathrm{x}}$ butenedial experiments are also similar, showing a sharp initial rise followed by a steady linear increase until the chamber is closed. The initial rise is smaller in the butenedial experiment but the subsequent increase occurs at much the same rate in both cases. Again this is different to the lower $\mathrm{VOC} / \mathrm{NO}_{\mathrm{x}}$ butenedial case where the ozone concentration-time profile is a curve that levels out at a peak concentration of about $325 \mathrm{ppb}$ before the chamber is closed. In contrast to the similar experimental concentration-time profiles, the simulated ozone profiles shown in Fig. 6 are very different from each other and from the observations. For the 4-oxo-2-pentenal experiment, the ozone profile is curved and levels out at a peak concentration of $458 \mathrm{ppb}$, over-predicting the observed ozone peak by $18 \%$, though the model-measurement discrepancy is much greater earlier in the experiment. For the high $\mathrm{VOC} / \mathrm{NO}_{\mathrm{x}}$ butenedial 
experiment, the simulated ozone is in excellent agreement with the measurements for the first hour and a half of the experiment but after that the simulated concentration rises significantly faster than the observations. After three hours, at which time the chamber closes, the ozone concentration is over-predicted by $87 \%$.

In the 4-oxo-2-pentenal and high $\mathrm{VOC} / \mathrm{NO}_{\mathrm{x}}$ butenedial experiments (Fig. 6) the observed $\mathrm{NO}_{2}$ concentrations peak sharply in the first half hour after the chamber opens. The initial NO concentration in the 4-oxo-2-pentenal case is around twice that in the high $\mathrm{VOC} / \mathrm{NO}_{\mathrm{x}}$ butenedial experiment and the peak $\mathrm{NO}_{2}$ is correspondingly higher, but in both cases the $\mathrm{NO}_{2}$ concentration falls to 8 or $9 \mathrm{ppb}$ approximately $50 \mathrm{~min}$ after the start of the experiment. In the high $\mathrm{VOC} / \mathrm{NO}_{\mathrm{x}}$ butenedial experiment, this $\mathrm{NO}_{2}$ concentration is maintained until the chamber is closed, while in the 4-oxo-2-pentenal experiment a steady increase in $\mathrm{NO}_{2}$ is observed until the concentration reaches about $19 \mathrm{ppb}$ and this is maintained until the chamber is closed. Different behaviour is seen for the lower $\mathrm{VOC} / \mathrm{NO}_{\mathrm{x}}$ butenedial experiment (Fig. 4), the $\mathrm{NO}_{2}$ concentration peaks early in the experiment then falls steadily throughout the experiment to about $10 \mathrm{ppb}$ when the chamber closes.

As discussed above for the low $\mathrm{VOC} / \mathrm{NO}_{\mathrm{x}}$ butenedial case, the observed $\mathrm{NO}_{2}$ concentrations are not well simulated by $\mathrm{MCMv3.1}$. In the simulation of the high $\mathrm{VOC} / \mathrm{NO}_{\mathrm{x}}$ butenedial experiment most of the $\mathrm{NO}_{2}$ initially present is removed and the $\mathrm{NO}_{2}$ concentration is very low for the first hour of the simulation before increasing to a peak of around $20 \mathrm{ppb}$ later in the experiment as the PAN type compounds decompose. In the 4-oxo-2-pentenal experiment, such a secondary $\mathrm{NO}_{2}$ peak is actually observed but not recreated in the simulations. The initial peak concentration is in good agreement with the measurement but the subsequent fall in concentration is slower than observed. After about two and a half hours the modelled concentration falls below the increasing measured concentration.

It is interesting to note that for the two lower $\mathrm{VOC} / \mathrm{NO}_{\mathrm{x}}$ experiments (4 July 2002 and 3 October 2001) if the $\mathrm{OH}$ concentration is constrained to the measurement the modelmeasurement comparisons for $\mathrm{NO}_{2}$ are greatly improved and the simulated ozone concentrations are in excellent agreement with the measurement. This is not the case for the higher $\mathrm{VOC} / \mathrm{NO}_{\mathrm{x}}$ butenedial experiment (2 October 2001) where constraining the $\mathrm{OH}$ to the high concentrations measured does not improve the agreement for $\mathrm{NO}_{2}$ and actually results in greater ozone formation and a final over-prediction of $160 \%$

\subsubsection{Photolysis experiments: in the absence of $\mathrm{NO}_{\mathrm{x}}$}

In addition to the photosmog experiments described above, a set of experiments on butenedial and 4-oxo-2-pentenal were carried out in the absence of $\mathrm{NO}_{\mathrm{x}}$ with initial $\gamma$-dicarbonyl concentrations of $250-300 \mathrm{ppb}$. In order to determine the radical yield of the photolysis of $\gamma$-dicarbonyls the $\mathrm{HO}_{2}$ concentration was measured by chemical conversion with $\mathrm{NO} /$ laser induced fluorescence. A detailed analysis of the experimental results will be presented by Thuener et al. (in preparation, 2005) ${ }^{1}$ and only a brief discussion of the performance of MCM in simulating these experiments is given here. One of the butenedial photolysis experiments is chosen as a representative case study, and similar conclusions can be drawn from analysis of the other experiments.

The MCMv3.1 simulations for butenedial, $\mathrm{HO}_{2}$ and some important products are compared to the measurements in Fig. 7. The simulation shown assumed the concentrations of $\mathrm{NO}$ and $\mathrm{NO}_{2}$ to be zero although it is not possible to have entirely $\mathrm{NO}_{\mathrm{x}}$ free conditions in the chamber as some residual off-gassing from the wall will always occur. However, measured $\mathrm{NO}_{2}$ concentrations were never more than $\sim 2 \mathrm{ppb}$ and at $\mathrm{NO}_{\mathrm{x}}$ levels of this order the simulations were not greatly changed from the $\mathrm{NO}_{\mathrm{x}}$ free case and the conclusions discussed below remain valid.

The loss rate of butenedial is well predicted which is a reflection of the reproducibility of the chamber experiments as the photolysis rate implemented in MCMv3.1 is derived from experiments very similar to this one. However, it is clear to see that, in contrast to the situation in the photosmog experiments, the $\mathrm{HO}_{2}$ concentration is over-estimated by the simulation by almost an order of magnitude early in the experiment. The time-dependent behaviour is also not well reproduced by the simulation as in the experiment an initial fast increase in concentration is followed by a slower linear increase until the chamber closes, while the simulation shows a fast rise followed by a fall in $\mathrm{HO}_{2}$ concentration even while the photolysis continues. The photolysis mechanism for butenedial in the absence of $\mathrm{NO}_{\mathrm{x}}$ as implemented in MCMv3.1 is shown schematically in Fig. 2. This indicates that two $\mathrm{HO}_{2}$ radicals should be formed for each molecule of maleic anhydride and glyoxal produced, and while both these product concentrations are over-estimated this is not sufficient to account for the large over-prediction of $\mathrm{HO}_{2}$. One must conclude that a different $\mathrm{HO}_{2}$ formation pathway is operating and/or a significant loss process for $\mathrm{HO}_{2}$ is not included in the mechanism. Thuener et al. (2003a) propose a different butenedial photolysis mechanism based on product experiments similar to the one considered here and the observation of an intermediate ketene species in all these experiments. However, that mechanism also requires two $\mathrm{HO}_{2}$ radicals per maleic anhydride molecule formed and similar problems arise when trying to account for the low concentration of $\mathrm{HO}_{2}$ observed.

Concerning the molecular products of butenedial photolysis, the yield of $2(5 \mathrm{H})$-furanone is well predicted by the

\footnotetext{
${ }^{1}$ Thuener, L. P., Rea, G., Wenger, J., Henderson, A. P., Bleasdale, C., and Golding, B. T.: Photolysis of Butenedial and 4oxopent-2-enal under atmospheric conditions, Environ. Sci. Technol., in preparation, 2005.
} 


\section{Butenedial (16/07/02) $\rightarrow$ experiment MCMv3.1}
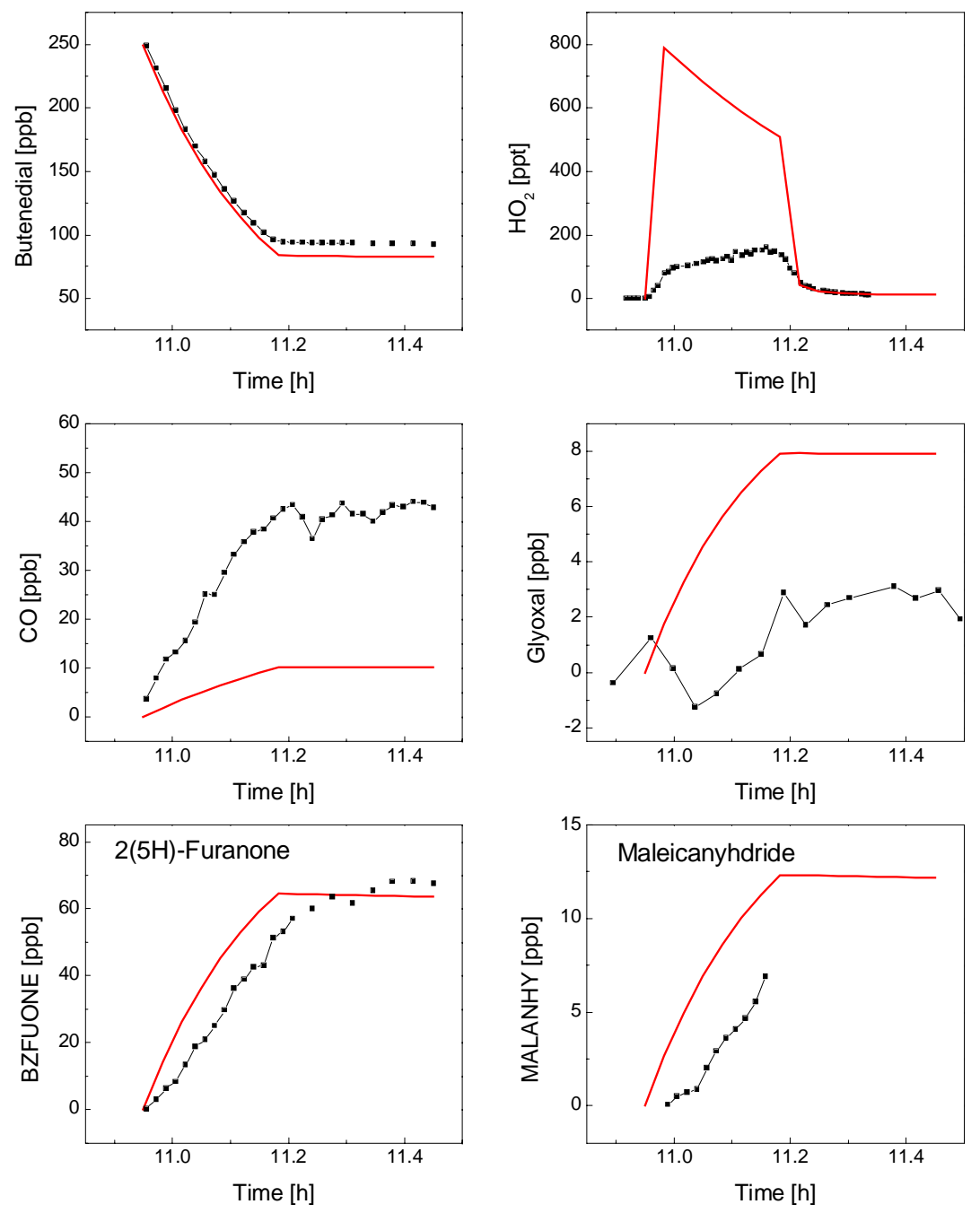

Fig. 7. Butenedial photolysis experiment in the absence of $\mathrm{NO}_{\mathrm{x}}$ (16 July 2002): comparison of modelled and measured concentrations of butenedial, $\mathrm{HO}_{2}$, and selected products.

simulation, glyoxal and maleic anhydride are over-predicted while the CO yield in the simulation is much lower than observed experimentally. In MCMv3.1 glyoxal and $\mathrm{CO}$ are formed as co-products, but this is not consistent with the different yields of these products observed experimentally. Thuener et al. (2003a) include a different source of CO in their proposed mechanism, i.e. direct formation from photolysis with a yield of $20 \%$. A possible co-product for direct $\mathrm{CO}$ production is acrolein formed by an $\mathrm{H}$ shift and $\mathrm{C}-\mathrm{C}$ cleavage. The acrolein concentration was below the detection limit of the measurement technique, and its maximum yield was estimated to be $\sim 10 \%$. No other direct photolysis products were observed and it was not possible to positively determine the mechanism and co-products for $\mathrm{CO}$ formation.
In addition to the products for which results are shown in Fig. 7, 4-Oxo-but-2-enoic acid and 4-Oxo-but-2-eneperoxoic acid are predicted by MCMv3.1 to be major products in the $\mathrm{NO}_{\mathrm{x}}$ free conditions of this experiment but were not observed. An unattributed carbonyl absorption was found in the FTIR spectra (Thuener et al., 2003a) but the absorption frequency of this unknown does not correspond to that expected for a conjugated carboxylic or peroxoic acid.

Although some improvements to our understanding of the photo-oxidation of $\gamma$-dicarbonyls have been made during the EXACT project the agreement between MCM simulations and chamber experiments both with and without $\mathrm{NO}_{\mathrm{x}}$ remains poor. Particular problems arise concerning the radical yields: seemingly contradictory behaviour is seen as 


\section{Cresol $(04 / 10 / 01)$}
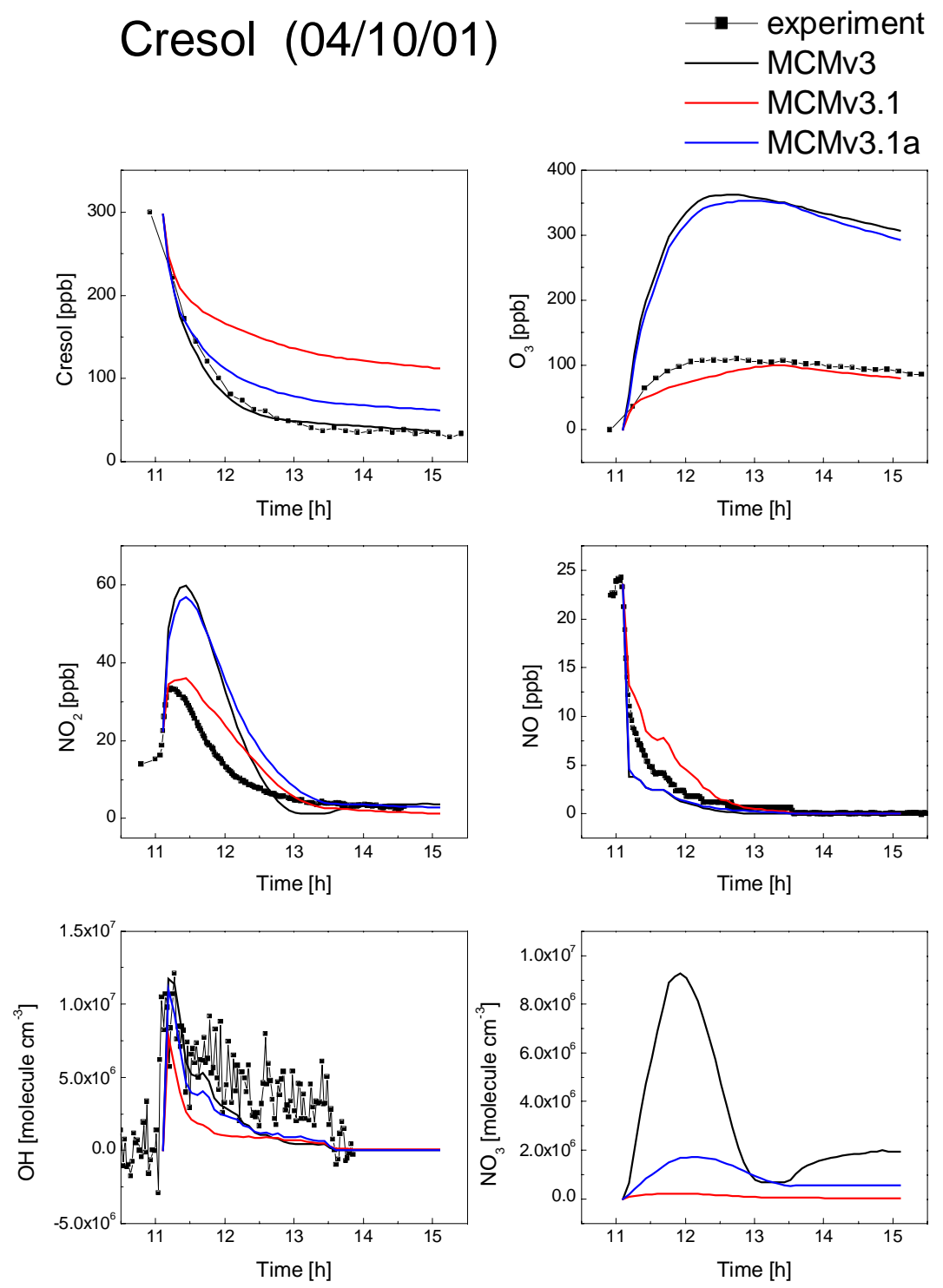

Fig. 8. Cresol photosmog experiment (4 October 2001); comparison of modelled and measured concentrations of cresol, $\mathrm{O}_{3}, \mathrm{NO}_{\mathrm{x}}$ and $\mathrm{OH}$ with MCMv3, MCMv3.1 and MCMv3.1a (see text). Also shown are modelled concentrations of $\mathrm{NO}_{3}$.

with $\mathrm{NO}_{\mathrm{x}}$ present the simulations under-estimate both $\mathrm{OH}$ and $\mathrm{HO}_{2}$ while in the absence of $\mathrm{NO}_{\mathrm{x}}$ the $\mathrm{HO}_{2}$ concentration is over-estimated. In the photosmog systems the $\mathrm{NO}_{\mathrm{y}}$ budget is not well understood and the simulations are unable to reproduce the different behaviours seen experimentally at different $\mathrm{VOC} / \mathrm{NO}_{\mathrm{x}}$ ratios and with different $\gamma$-dicarbonyls. It is therefore difficult to identify mechanistic changes which can simultaneously resolve the discrepancies seen in the two systems (i.e. photosmog and photolysis), based on current general understanding of processes involved in free radical driven VOC oxidation. Nevertheless, overcoming these shortcomings in an area of the mechanism identified as being of key importance in determining ozone and $\mathrm{OH}$ concentrations is an important issue for improving our under- standing of aromatic chemistry. The EUPHORE chamber dataset on unsaturated $\gamma$-dicarbonyls provides an important resource for testing future mechanism developments and the work presented here highlights issues that require further investigation.

\subsection{Photo-oxidation of cresol}

The initial conditions for the cresol photosmog experiment are summarised in Table 3 , and the measured and simulated concentrations of the key species in this system are shown in Fig. 8.

MCMv3 gives an excellent representation of the loss of cresol but over-predicts the ozone formation by more than a factor of 3. It should be noted that, in contrast to the aromatic 
hydrocarbons where $\mathrm{OH}$ determines the loss rate, cresol reacts with both $\mathrm{OH}$ and $\mathrm{NO}_{3}$. The comparison of modelled and measured $\mathrm{OH}$ concentration shows that $\mathrm{MCMv} 3$ underpredicts $\mathrm{OH}$ in the later stages of the experiment, and the observed agreement concerning the loss of cresol is a result of the over-prediction of $\mathrm{NO}_{3}$ concentrations.

The observation of high ring-retained product yields from cresol oxidation (Olariu et al., 2002) led to the development of a provisional revised mechanism, designated MCMv3.1a in Fig. 8. The first stage of this mechanism is identical to MCMv3.1 and is described in detail in Sect. 2.4. The major product of the $\mathrm{OH}$ initiated chemistry is 1,2-dihydroxy3-methyl benzene, and nitrocresol is formed in both the $\mathrm{OH}$ and $\mathrm{NO}_{3}$ initiated chemistry. The yields of these products are assigned from the experimental evidence. The first generation ring-retained products are then treated in the same way as the original cresol, i.e. assuming the same branching ratios for the different $\mathrm{OH}$ and $\mathrm{NO}_{3}$ initiated channels. The second generation ring-retained products are then assumed to undergo ring-opening. This chemistry is illustrated in Fig. 3.

Despite the extensive changes to the mechanism, it is clearly shown in Fig. 8 that this new version, MCMv3.1a, over-predicts ozone formation by a similar amount to $\mathrm{MCMv3}$. The major products are dihydroxy- and trihydroxysubstituted toluene and these have very short lifetimes as $\mathrm{OH}$ groups activate the aromatic ring. The result is the formation of ring-opened products that act as radical sources and lead to ozone production. The simulated $\mathrm{NO}$ to $\mathrm{NO}_{2}$ conversion is faster than experimentally observed and the $\mathrm{NO}_{2}$ concentration is significantly over-predicted.

Following the identification of these shortcomings of the cresol mechanism when simulating chamber data, adjustments were made to improve the model-measurement agreement. There is no information available on the product yields from the oxidation of 1,2-dihydroxyarenes and hydroxynitroarenes, and the mechanism was altered so that reaction of $\mathrm{OH}$ and $\mathrm{NO}_{3}$ with these products is assumed to proceed solely by formation of phenoxy radicals that react with $\mathrm{NO}_{2}$ adding a nitro- group to the aromatic ring. The active pathways in the $\mathrm{OH}$ initiated chemistry of MCMv3.1 are shown in black in Fig. 3, and it is clear that the mechanism is a simpler version of MCMv3.1a. The second generation of products from hydroxyarene oxidation are 1,2-dihydroxynitroarenes and dinitro-hydroxyarenes. Further reaction of $\mathrm{OH}$ and $\mathrm{NO}_{3}$ is assumed to proceed via peroxide-bicyclic channels leading to ring-opening and formation of representative hydroxy- and nitro-substituted $\alpha$-dicarbonyls and $\alpha$, $\beta$-unsaturated- $\gamma$-dicarbonyls. The multi-substituted bicyclic radicals are representative examples of possible degradation pathways. The products were chosen to simplify the subsequent chemistry and these channels may not be strictly available from the bicyclic alkoxy radicals.

Addition of an $\mathrm{NO}_{2}$ group deactivates the aromatic ring and the nitro- substituted cresols have longer lifetimes than dihydroxy- and trihydroxy- substituted toluene. Increasing the relative yields of these nitro- compounds therefore decreases the amount of ring-opening that occurs in the timescale of the chamber experiment and reduces the radical production. The formation of nitro- compounds also acts as a $\mathrm{NO}_{2}$ sink and reduces the simulated $\mathrm{NO}_{2}$ concentration. The concentrations of key species simulated with the MCMv3.1 mechanism are shown in red in Fig. 8. The production of ozone is dramatically reduced compared to the previous versions and is in good agreement with the measurement. As expected, the simulated $\mathrm{NO}_{2}$ concentration has been reduced and $\mathrm{NO}$ to $\mathrm{NO}_{2}$ conversion is now slower than the measurements suggest. The reduction in simulated radical concentration is evident in the under-prediction of measured $\mathrm{OH}$ and loss rate of the parent cresol compound.

This under-estimation of oxidative capacity is also seen in the simulations using the toluene mechanism, of which this cresol mechanism is a part. Ideas for solving this problem while not concurrently increasing ozone production are discussed below in Sect. 4.4.

Concentrations of key intermediate species were also measured in the chamber experiment. Glyoxal is formed in the ring-opening route and its measured peak concentration in this experiment is $5.5 \mathrm{ppb}$. MCMv3 assumes ring-opening to be the major oxidation process for cresol and the simulated peak glyoxal concentration is $137 \mathrm{ppb}$, a factor of 25 greater than observed. The reduced channel yield in MCMv3.1 reduces the simulated peak glyoxal concentration to $13.5 \mathrm{ppb}$, the model still over-predicts the observed concentration but the agreement is greatly improved. The ring retained product 1,2-dihydroxy-3-methyl benzene is not included in MCMv3 but is the major product in the $\mathrm{OH}$ initiated cresol oxidation as implemented in MCMv3.1. The simulated peak concentration is $30 \mathrm{ppb}$. The chamber measurements confirm this compound as a product, and the observed peak concentration is $12 \mathrm{ppb}$.

\subsection{Carbon budget in toluene oxidation}

There have been many studies of the products of $\mathrm{OH}$-initiated toluene oxidation and an overview of the available product data is given by Calvert et al. (2002). However, all these product studies show a deficient carbon balance with generally only about $50 \%$ of the reacted carbon being identified as products. Particular uncertainties exist in the ring-opening routes, e.g. the peroxy-bicyclic and epoxy routes of Fig. 1. The $\alpha$-dicarbonyl species glyoxal and methylglyoxal are well known ring-opened products of toluene oxidation with yields determined in various studies. Recent work by Volkamer et al. (2001) has quantified the primary glyoxal yield from toluene oxidation and found the secondary yield to be negligible. This is important information for assigning the channel yield for the peroxy-bicyclic channel but the identity and yields of the co-products of $\alpha$-dicarbonyls are highly uncertain. Reactive unsaturated dicarbonyl compounds have been previously observed in the oxidation of toluene but not 
quantified. As discussed above in Sect. 2.1, the $\mathrm{O}_{3}$ and $\mathrm{OH}$ concentrations in the toluene photosmog system have been found to be sensitive to the concentrations of such intermediates, and their quantification is necessary to form a good understanding of aromatic oxidation and its effect on ozone formation in the troposphere.

$\mathrm{GC} \times \mathrm{GC}$ and GC-ECD techniques were used to detect products from toluene oxidation in the first measurement campaign held at EUPHORE in 2001, and provided some qualitative evidence for the formation of compounds such as butenedial, 4-oxo-2-pentenal and $\alpha$-angelicalactone. Table 4 shows the results obtained on these key intermediates. However, quantification was difficult and experimentally observed concentrations were much lower than predicted by the models (Hamilton et al., 2003).

Experiments on toluene photo-oxidation designed to maximise the concentrations of the co-products of $\alpha$-dicarbonyls were carried out during the second measurement campaign. High initial concentrations of toluene $(\sim 6 \mathrm{ppm})$ and $\mathrm{NO}_{\mathrm{x}}$ $(500 \mathrm{ppb})$ were used and the chamber was closed at the point in the experiment where maximum concentrations of butenedial and 4-oxo-2-pentenal are expected, thus preventing loss by photolysis and $\mathrm{OH}$ reaction and keeping the concentrations high for detection by analytical methods. The simulations suggest that this approach should result in peak concentrations of these compounds approximately a factor of 10 higher than in the experimental conditions of the first campaign and this concentration should be maintained within $30 \%$ of the peak value for more than one hour after closing the chamber.

High, and sustained, concentrations of $\alpha$-dicarbonyl compounds were measured in these experiments demonstrating the utility of this approach for measurement of intermediates. For example, in the first two toluene experiments (5 and 10 July 2002) the measured peak methylglyoxal concentrations were $70 \mathrm{ppb}$ and $47 \mathrm{ppb}$, respectively; these concentrations were measured as the chamber closed and subsequently decreased by only about $10 \%$ in the following two hours. The peak concentrations were within $15 \%$ of those simulated using MCMv3.1 and lead us to expect peak concentrations of the co-product, butenedial, of $14 \mathrm{ppb}$ and $11 \mathrm{ppb}$ respectively. The initial toluene concentration was increased for the experiment on 12 July 2002 to further increase the product concentrations. In this case the model over-predicts the initial reactivity of the system and hence over-predicts the observed methylglyoxal concentration by a factor of two. Taking into account this over-prediction a peak butenedial concentration of $17.5 \mathrm{ppb}$ was expected. However, only small amounts of furanone and $\gamma$-dicarbonyl compounds were observed by $\mathrm{GC} \times \mathrm{GC}$ and GC-ECD techniques in these experiments. After the chamber was closed on 10 July 2002, $50 \mathrm{ppb}$ of butenedial was introduced into the chamber to calibrate the GC instruments in the presence of interferences from other reaction products. The chamber was then flushed to dilute the contents before taking further measurements, and an upper
Table 4. Measurement of ring-opening products in the toluene system during the EXACT-1 campaign $(<\mathrm{LOD}=$ concentration below limit of detection, NT=not targeted).

\begin{tabular}{|c|c|c|}
\hline $\begin{array}{l}\text { Putative intermediates in } \\
\text { ring-opening pathways }\end{array}$ & $\mathrm{GC} \times \mathrm{GC}$ & GC-ECD \\
\hline 4-Oxo-2-pentenal & Detected & Detected \\
\hline Butenedial & $<$ LOD & Detected \\
\hline a-Angelicalactone & Detected & Detected \\
\hline 2(5H)-Benzofuranone & $<$ LOD & NT \\
\hline Maleic anhydride & Detected & Detected \\
\hline Methylmaleic anhydride & NT & Detected \\
\hline 6-Oxo-2,4-heptenal & $<$ LOD & NT \\
\hline 2,3-Epoxybutanal & $<$ LOD & NT \\
\hline Number of unidentified signals & 2 & \\
\hline
\end{tabular}

limit of $2 \mathrm{ppb}$ was estimated for the concentration of butenedial observed by GC-ECD during this experiment. A similarly sized signal was observed in the 12 July 2002 experiment. The furanone and $\gamma$-dicarbonyl compounds are difficult to measure but the observations suggest that they were not formed in the relatively high concentrations predicted by the model.

Despite the low amounts of unsaturated dicarbonyl and furanone compounds observed in these experiments the compounds are included as co-products of glyoxal and methylglyoxal in the MCM as no alternative products could be observed. Based on our understanding of the formation mechanism for $\alpha$-dicarbonyls (Jenkin et al., 2003) some products of this type must be formed, and if butenedial, 4-oxo-2-pentenal and $\alpha$-angelicalactone are not formed in the yields expected they can be considered as models for the true co-products.

\subsection{Shortcomings of the aromatic mechanisms}

In the companion paper (Bloss et al., 2005) the performance of MCMv3 and MCMv3.1 aromatic mechanisms are evaluated using the EUPHORE chamber dataset on benzene, toluene, $p$-xylene and 1,3,5-trimethylbenzene. Although MCMv3.1 simulations give an improved representation of some of the observations, significant discrepancies remain. Of particular importance to modelling regional air quality, the mechanisms tend to over-predict the peak ozone concentration reached. However, at the same time the mechanisms under-predict the reactivity of the system with lower concentrations of $\mathrm{OH}$ produced than are required to explain the observed loss rate of the parent hydrocarbons and lower NO oxidation rates than seen experimentally. Any reduction of ozone production by reduction of peroxy radical concentration to limit $\mathrm{NO}$ to $\mathrm{NO}_{2}$ conversion will also result in decreased $\mathrm{OH}$ production and $\mathrm{NO}$ oxidation rate and hence greater model-measurement discrepancy in these 


\section{Toluene $(27 / 09 / 01)$}
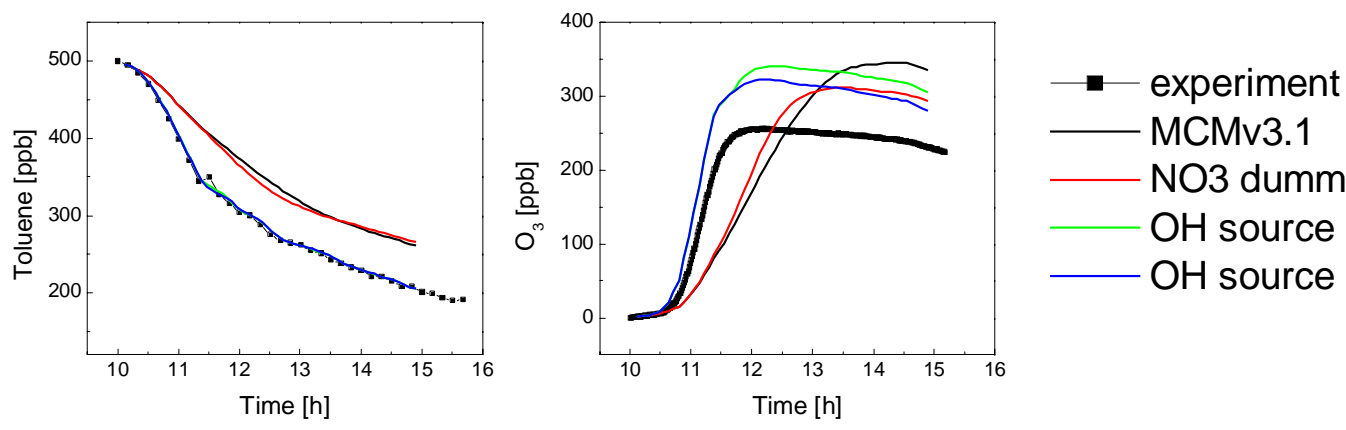

- MCMv3.1

NO3 dummy reaction

$\mathrm{OH}$ source

- $\mathrm{OH}$ source and $\mathrm{NO} 3$ dummy
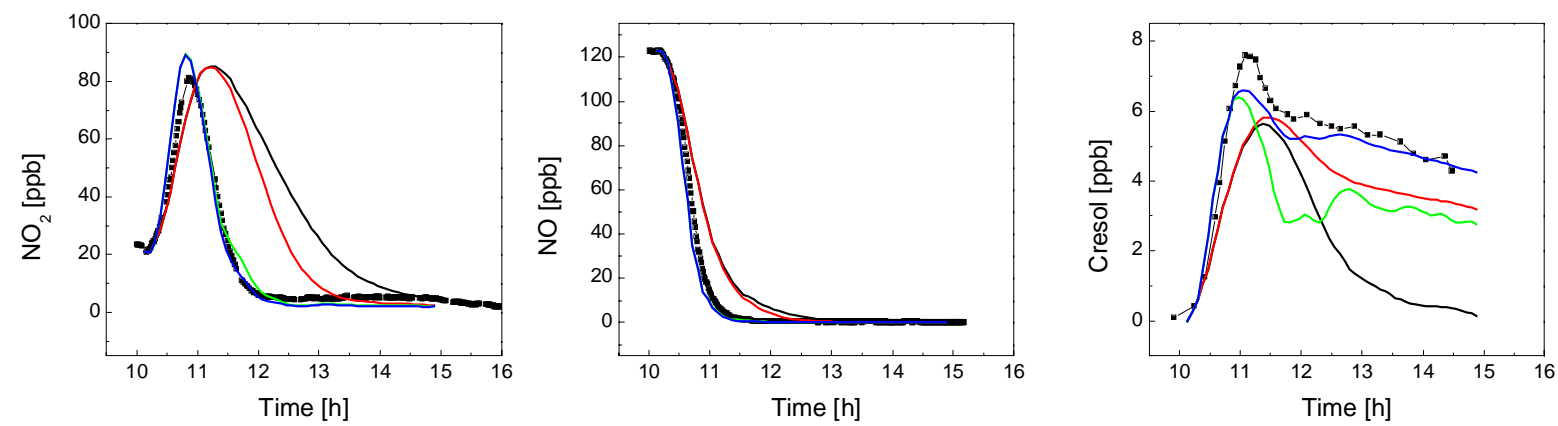

Fig. 9. Toluene experiment (27 September 2001); comparison between experimental and simulated concentration time-profiles for MCMv3.1, mechanism with a dummy $\mathrm{NO}_{3}$ conversion to $\mathrm{HO}_{2}$ included and mechanisms with artificial $\mathrm{OH}$ source added in order to reproduce toluene loss.

areas. These effects are also seen in the simulations of the cresol chamber experiment discussed above in Sect. 4.2.

The results of a simulation where an artificial source of $\mathrm{OH}$ was added to the model for the moderate $\mathrm{NO}_{\mathrm{x}}$ toluene experiment, such that the modelled toluene loss rate equals the observed rate, are shown in green in Fig. 9, and this can be compared to the MCMv3.1 simulation in black. The $\mathrm{OH}$ source is active throughout the experiment and the average rate of $\mathrm{OH}$ production is $4 \times 10^{8}$ molecule $\mathrm{cm}^{-3} \mathrm{~s}^{-1}$. As discussed in the companion paper (Bloss et al., 2005), such a large radical source cannot be accounted for by the chamber auxiliary mechanism and must be connected to the chemistry of the aromatic photosmog system. The additional $\mathrm{OH}$ in the simulation greatly improves the model-measurement comparison for $\mathrm{NO}$ and $\mathrm{NO}_{2}$, improves the agreement with the initial rate of ozone production, but continues to overestimate the peak ozone concentration.

It is important to assess the statistical significance of the discrepancy between the measurements and the models. This has been done extensively in recent papers. Zador et al. (2004) carried out a detailed statistical analysis for ethene photosmog experiments, examining uncertainties in both models and measurements. Bloss et al. (2003) made a careful comparison of the rate of decay of several aromatics and $[\mathrm{OH}]$ as measured by FAGE and found generally good agreement, within the combined uncertainty limits. It is clear that the discrepancies in ozone, $\mathrm{OH}$ and $\mathrm{NO}_{\mathrm{x}}$ concentrations found in the present papers far exceed those expected on grounds of measurement and in model parameter uncertainties. Hamilton et al. (2003) used GC $\times$ GC to search for the expected co-products of glyoxal and methyl glyoxal in toluene, but the yields were substantially below those predicted by the current mechanism.

A number of ideas have been explored to account for the observed model shortcomings, and these are summarised below, using the moderate $\mathrm{NO}_{\mathrm{x}}$ toluene experiment $(27$ September 2001) as an example.

\subsubsection{Regeneration of $\mathrm{OH}$}

One means of decreasing ozone production while increasing $\mathrm{OH}$ concentrations is to implement a regeneration of $\mathrm{OH}$ without conversion of $\mathrm{NO}$ to $\mathrm{NO}_{2}$. In the peroxide-bicyclic route of the MCM, a peroxy radical is formed by addition of $\mathrm{OH}$ and two $\mathrm{O}_{2}$ molecules to the aromatic ring; this then reacts with $\mathrm{NO}$ to form an alkoxy radical (and $\mathrm{NO}_{2}$ ), which decomposes to ring-opened products, and $\mathrm{HO}_{2}$. A further $\mathrm{NO}$ to $\mathrm{NO}_{2}$ conversion $\left(\mathrm{HO}_{2}\right.$ reaction) produces $\mathrm{OH}$. Figure 10 shows, in red, the effect on the simulations of replacing the usual peroxy radical chemistry by a direct decomposition to ring-opened products and $\mathrm{OH}$. This could occur following a 

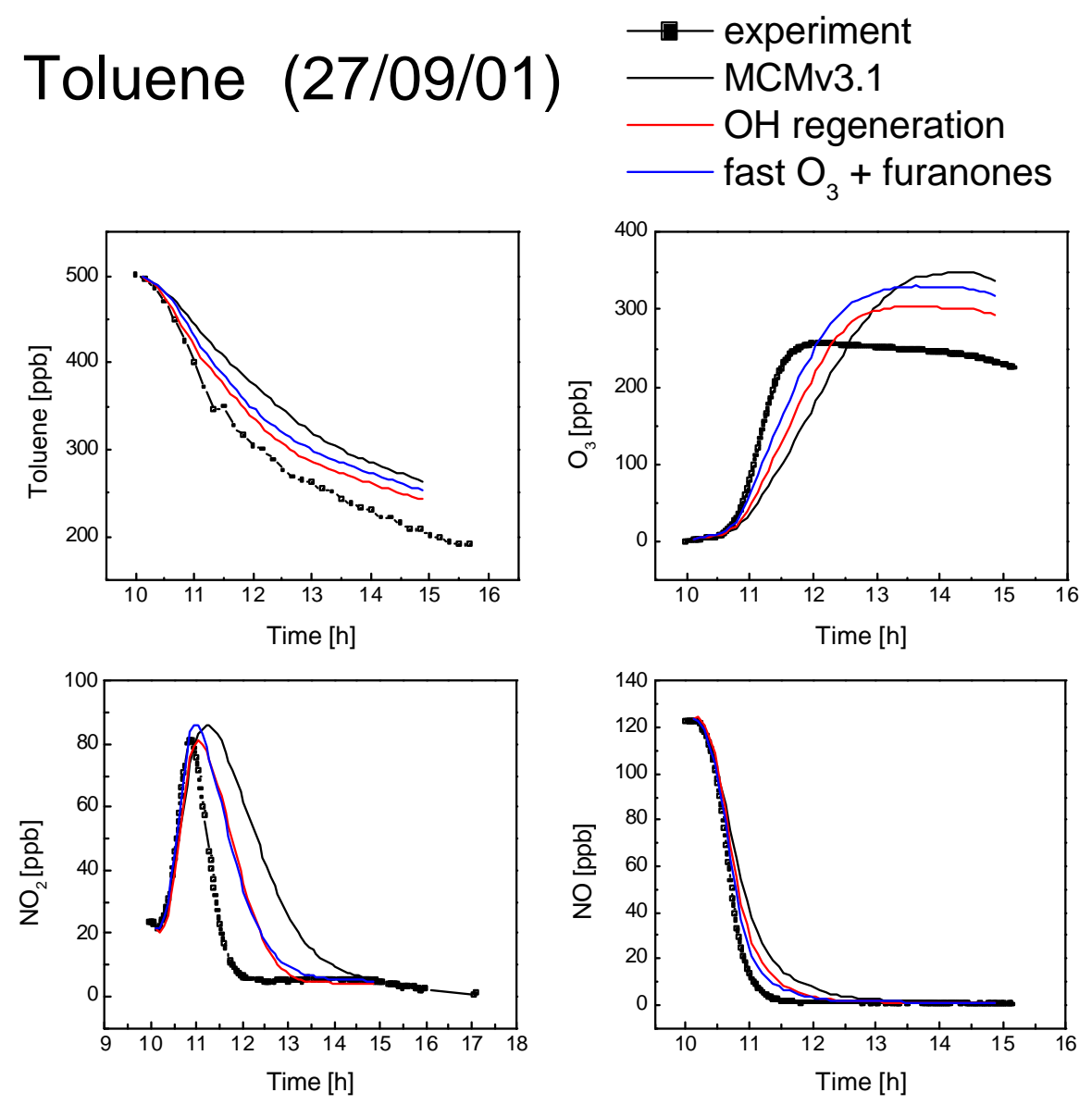

Fig. 10. Toluene experiment (27 September 2001); comparison between experimental and simulated concentration time-profiles for MCMv3.1 (black), a mechanism including a speculative $\mathrm{OH}$ regeneration mechanism in the peroxide-bicyclic route (red), and the MCMv3.1 mechanism but with rate coefficients for $\mathrm{O}_{3}$ +furanones of $3.5 \times 10^{-15} \mathrm{~cm}^{3}$ molecule $^{-1} \mathrm{~s}^{-1}$ (blue).

rearrangement of the peroxy radical by an $\mathrm{H}$-shift, as indicated in Fig. 11.

It is clear to see that this alteration in the mechanism improves the model-measurement agreement. The peak ozone is decreased by $12 \%$ compared to MCMv3.1, and the measured peak concentration is over-predicted by $19 \%$ by the modified mechanism, $36 \%$ by MCMv3.1. At the same time the modelled loss of toluene is faster than in MCMv3.1 as a result of higher $\mathrm{OH}$ concentrations, giving a better representation of the observations but still an under-estimate of the reactivity of the system.

\subsubsection{Ozonolysis of intermediate compounds}

A fast reaction of ozone with one or more of the intermediates in the system to produce radicals would result in lower ozone concentrations and an increase in $\mathrm{OH}$. The effect of increasing the ozonolysis rate coefficient for the furanone compounds to $3.5 \times 10^{-15} \mathrm{~cm}^{3}$ molecule ${ }^{-1} \mathrm{~s}^{-1}$, as a model for this type of chemistry, is shown in blue in Fig. 10. A rate coefficient of this magnitude has been measured for 4,5 dihydro-2-methyl furan (Martin et al., 2002), but the measurements of Grosjean and Grosjean (1999) discussed above in Sect. 2.3 suggest a slow ozonolysis rate for the furanones. However, the precise nature of the intermediates produced in the aromatic systems remain uncertain, and one or more of them may have a fast reaction with ozone.

Model-measurement agreement is improved by the modification to the mechanism, but peak ozone concentration is still over-predicted by $29 \%$. Increased $\mathrm{OH}$ concentrations lead to a faster loss of toluene but, as with the $\mathrm{OH}$ regeneration mechanism, the reactivity of the system remains underestimated.

\subsection{3 $\quad \mathrm{NO}_{3}$ chemistry}

As the production of ozone in photosmog systems is closely connected to the $\mathrm{NO}_{\mathrm{x}}$ chemistry another strategy for decreasing ozone formation while increasing reactivity is to include a process acting as both a sink for $\mathrm{NO}_{\mathrm{x}}$ and a source of 


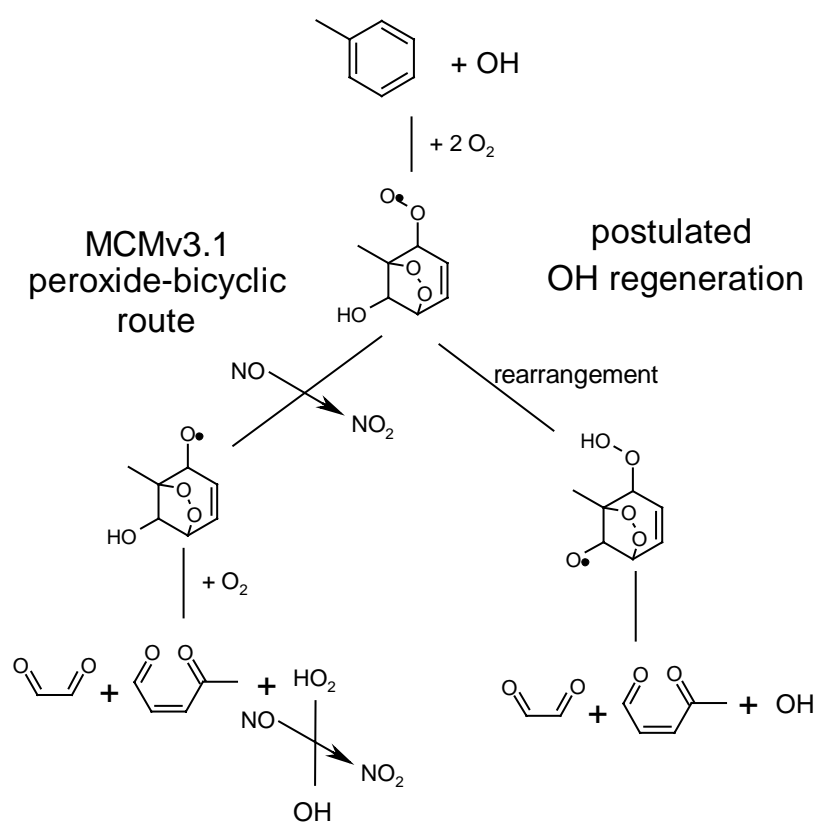

Fig. 11. Schematic of MCMv3.1 peroxide bicyclic route for toluene oxidation and postulated $\mathrm{OH}$ regeneration pathway.

$\mathrm{HO}_{\mathrm{x}}$ radicals. The nitrate radical, $\mathrm{NO}_{3}$, is closely coupled to $\mathrm{NO}$ and $\mathrm{NO}_{2}$ chemistry, and $\mathrm{NO}_{3}$ can react with compounds such as aldehydes and unsaturated compounds forming radical products. The reactions of $\mathrm{NO}_{3}$ are included in the mechanism where appropriate, though often with generic rate coefficients and product distributions estimated by analogy.

It was noted in the companion paper (Bloss et al., 2005) that the loss rate of cresol product is over-estimated by the models in the toluene experiments and this is a result of an over-prediction of $\mathrm{NO}_{3}$ concentration. The measured concentration-time profile of cresol can be used as a tracer for $\mathrm{NO}_{3}$ concentration in the experiment

A dummy reaction was added to the model to represent the reaction of $\mathrm{NO}_{3}$ with unknown compounds producing $\mathrm{HO}_{2}$. This reaction was assigned a rate coefficient of $2 \times 10^{-12}$ molecule ${ }^{-1} \mathrm{~cm}^{3} \mathrm{~s}^{-1}$ and the concentration of the reactant was assumed to be constant at $100 \mathrm{ppb}$. While this is clearly an over-simplification and no account is taken of the onward chemistry of the other products of this hypothetical $\mathrm{NO}_{3}$ reaction, it does serve to demonstrate the effect of such a process in the mechanism.

The result of this modification to the mechanism is shown in red in Fig. 9 compared to MCMv3.1 in black. The reduction of $\mathrm{NO}_{3}$ concentration is shown by the slower cresol decay around midday, the $\mathrm{NO}_{2}$ concentration has been reduced in the later stages of the experiment and the peak ozone concentration is also lower than in MCMv3.1. The toluene loss rate is slightly increased in the middle of the experiment but decreased at the end of the experiment, there is not a great improvement in the model-measurement agreement concerning the reactivity of the system.

Also shown in Fig. 9 are the results of simulations where an artificial source of $\mathrm{OH}$ was added to the model such that the modelled toluene loss rate equals the observed rate. As described above, for the concentration-time profiles shown in green this was the only modification to MCMv3.1. The additional $\mathrm{OH}$ in the simulation improves the agreement with the cresol concentration-time profile although the loss rate between 11 and 12 o'clock is still too high. The final set of simulations in Fig. 9 (in blue) show the result of adding both an $\mathrm{OH}$ source and the $\mathrm{NO}_{3}$ dummy reaction to the mechanism. The cresol profile is then well simulated, indicating agreement concerning $\mathrm{NO}_{3}$ concentrations, the model-measurement agreement for $\mathrm{NO}_{2}$ has been further improved around midday and the peak ozone concentration is decreased.

These simulations show that some $\mathrm{NO}_{3}$ chemistry may be missing from the mechanism and could help to explain some of the model-measurement discrepancies. The $\mathrm{NO}_{3}$ could be reacting with compounds that are not currently included in the mechanism, or the rates and products of some $\mathrm{NO}_{3}$ reactions in the mechanism may be incorrect. However, any such modifications will not solve the problem of the underestimation of the oxidative capacity in the system.

\subsubsection{Heterogeneous chemistry}

In the aromatic chamber experiments, aerosol particles are formed as a result of the production of semi-volatile and involatile oxidation products which are able to transfer to the condensed phase (Kalberer et al., 2004). Because the aerosol yields in such systems are generally quite low, it is likely that impact of loss of organic material to the condensed phase has only a small direct effect on the gas phase organic chemistry. This issue is discussed at greater length in the companion paper. However, the particles also potentially provide a substrate for heterogeneous reactions of both inorganic and organic species which might have an impact on the system. One such example is the heterogeneous conversion of $\mathrm{NO}_{2}$ to HONO, which has been observed to occur on soot particles (e.g. Arens et al., 2001 and references therein), and on diesel exhaust particulates (Gutzwiller et al., 2002). In the latter study, it was suggested that key surface-bound reactive components are species such as guaiacol (2-methoxyphenol) and other oxygenated aromatics, which are able to reduce $\mathrm{NO}_{2}$ to HONO. It is likely that the secondary organic aerosol formed in the present chamber experiments contains similar species (e.g. some of the second generation products of the "phenol" route identified in Fig. 1), and it is therefore of relevance to consider the potential impact of such a reaction. In the illuminated chamber, the formation and subsequent photolysis of HONO has the overall effect of producing $\mathrm{OH}$ radicals, and converting $\mathrm{NO}_{2}$ to $\mathrm{NO}$, which essentially equates to removing ozone. 


\section{Toluene $(27 / 09 / 01)$}
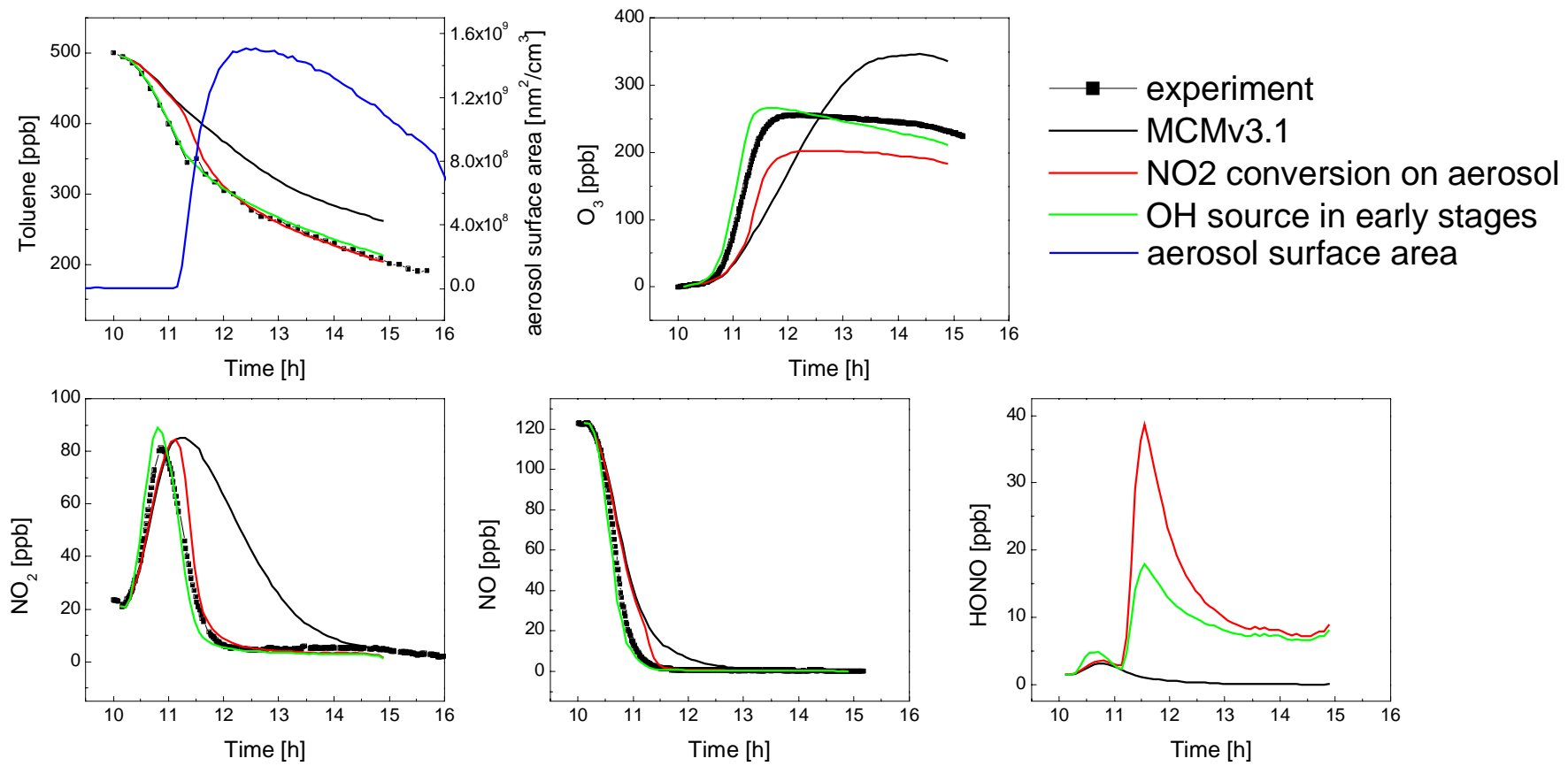

Fig. 12. Toluene experiment (27 September 2001); comparison between experimental and simulated concentration time-profiles for MCM3.1 and mechanisms including a conversion of $\mathrm{NO}_{2}$ to $\mathrm{HONO}$ on the aerosol surface. The measured aerosol surface area as a function of time is shown in blue.

The time dependent particle concentration and size distributions were measured using a scanning mobility particle sizer (SMPS), and from this information a rate of $\mathrm{NO}_{2}$ reaction on the aerosol could be calculated as a function of time, assuming a constant reaction probability over time and aerosol size. In Fig. 12 the simulated concentration-time profiles, assuming a reaction probability of 0.025 , are shown in red. The total surface area of the aerosol is also shown indicating that a sharp onset of particle formation was observed just after 11 a.m. At this point the simulations show a decrease in $\mathrm{NO}_{2}$ concentration and increase in HONO concentration. The $\mathrm{OH}$ concentration also increases rapidly, as evidenced by the increase in toluene loss rate. The peak ozone concentration is decreased and under-estimates the measured value. However, this under-estimation is a result of the low reactivity in the early part of the simulation before aerosol formation takes place. If an $\mathrm{OH}$ source is introduced to reproduce the toluene loss in the first hour of the simulation the $\mathrm{NO}_{\mathrm{x}}$ and $\mathrm{O}_{3}$ concentrations agree well with the measurements, as shown in green in Fig. 12.

The operation of such a heterogeneous process could clearly help to explain the model-measurement discrepancy for the parent aromatic, $\mathrm{NO}_{\mathrm{x}}$ and ozone, through increasing radical formation whilst simultaneously reducing the peak ozone concentration. However, while the simulations predict high peak HONO concentrations, no evidence for HONO formation was seen in the FTIR spectra suggesting that the concentration was below approximately $6 \mathrm{ppb}$. This indicates that any significant reaction of $\mathrm{NO}_{2}$ with the aromatic secondary organic aerosol formed in the chamber cannot lead to substantial formation of HONO in the gas phase, such that the reaction would need to generate $\mathrm{OH}$ and $\mathrm{NO}$ directly to have the desired effect. In addition, recent studies of the reactivity of $\mathrm{NO}_{2}$ with a variety of secondary organic aerosols (Bröske et al., 2003) have provided no evidence for reaction (although it is noted that the compositions of the aerosols almost certainly differ from those generated in the present chamber experiments). Consequently, the current weight of evidence suggests that the reaction of $\mathrm{NO}_{2}$ with organic aerosol does not account for the observed model shortcomings.

Simulations were also carried out to test the possibility of a reaction of $\mathrm{O}_{3}$ with the aerosol to produce $\mathrm{OH}$ radicals. These did not agree well with the measurements as too much ozone is removed in the later stages of the experiment where aerosol concentrations are high. 


\section{Conclusions}

The detailed oxidation mechanisms of aromatic hydrocarbons in the MCM have been revised and updated based on the latest available experimental data. Particularly important developments include the adjustment of branching ratios for the different oxidation routes of aromatics to reflect the reported yields of glyoxal and of phenol-type compounds at $\mathrm{NO}_{\mathrm{x}}$ levels appropriate to the atmosphere (Volkamer et al., 2001, 2002), and the implementation of new hydroxyarene degradation chemistry reflecting the high yields of ring-retained products reported by Olariu et al. (2002).

A series of chamber experiments were carried out to investigate the details of key areas of aromatic oxidation mechanisms, and these were primarily focused on toluene oxidation. Sensitivity analysis has identified important species affecting simulated $\mathrm{OH}$ and $\mathrm{O}_{3}$ concentrations and these include the $\gamma$-dicarbonyl intermediates formed in the ringopening routes (Wagner et al., 2003). Comparison of modelled and measured concentrations from photosmog experiments on butenedial and 4-oxo-2-pentenal indicated a number of shortcomings in the mechanisms. $\mathrm{NO}_{2}$ concentrationtime profiles are poorly simulated for all experiments, and the differences between experiments under different initial conditions are not well represented. It is clear that the $\mathrm{NO}_{\mathrm{xy}}$ budget is not well understood. The simulated $\mathrm{OH}$ and $\mathrm{HO}_{2}$ radical concentrations are significantly lower than the observed values and it seems that a large radical source is missing from the mechanism. However, very low $\mathrm{HO}_{2}$ radical concentrations were measured in $\gamma$-dicarbonyl photolysis experiments in the absence of $\mathrm{NO}_{\mathrm{x}}$; these measured concentrations were much lower than $\mathrm{HO}_{2}$ concentrations simulated by MCMv3.1, and this important issue remains unresolved.

The photo-oxidation of $o$-cresol was investigated in a separate chamber experiment and the results were used to adjust the representation of hydroxyarene degradation in MCMv3.1. The peak $\mathrm{O}_{3}$ concentration is well simulated by MCMv3.1, and the representation of the $\mathrm{NO}$ and $\mathrm{NO}_{2}$ concentration-time profiles is improved from MCMv3. However, the simulated radical yield is too low and the rate of cresol oxidation is therefore underestimated.

These model shortcomings confirm the mechanistic difficulties identified in the complete aromatic mechanisms in the companion paper (Bloss et al., 2005). In particular, the models tend to over-predict peak $\mathrm{O}_{3}$ concentrations, and at the same time under-estimate the NO oxidation rate and the overall oxidative capacity of the aromatic systems.

A number of possible mechanistic fixes have been investigated.

- An increase in $\mathrm{OH}$ yield without additional $\mathrm{NO}$ to $\mathrm{NO}_{2}$ conversion would improve the simulated concentrationtime profiles. A potential $\mathrm{OH}$ regeneration channel has been identified, but there is no firm evidence for its occurrence.
- A fast reaction of $\mathrm{O}_{3}$ with an intermediate to produce $\mathrm{OH}$ would result in improved model-measurement agreement. However, a very large rate coefficient and substantial intermediate concentrations would be required to give good agreement.

- Conversion of $\mathrm{NO}_{3}$ to $\mathrm{HO}_{2}$ by a dummy reaction in the model decreases $\mathrm{O}_{3}$ yields but does not significantly affect the aromatic decay rate.

- A modelled conversion of $\mathrm{NO}_{2}$ to $\mathrm{HONO}$ on the secondary organic aerosol has been shown to improve model-measurement agreement for $\mathrm{O}_{3}, \mathrm{NO}_{\mathrm{x}}$ and toluene decay. However, the relatively high HONO concentrations generated have not been observed experimentally, and the reactive uptake coefficient required is much higher than the upper limits for such processes suggested by the work of Bröske et al. (2003) for a variety of secondary organic aerosols.

A number of experimental strategies for resolving some of these issues can be proposed.

More experiments are needed to determine the yields of reactive oxygenated intermediates formed in aromatic degradation. In particular, it is essential to identify quantitatively the co-products of glyoxal and methyl glyoxal in toluene oxidation. This is a demanding requirement, since the species formed are likely to be highly photochemically reactive. Ideally these would be carried out with high time resolution as an aid to distinguishing primary and secondary oxidation products. Sensitive, on-line analytical techniques should be employed to detect and quantify such intermediates in chamber experiments carried out under $\mathrm{NO}_{\mathrm{x}}$ conditions representative of atmospheric levels. Proton-transfer-reaction mass spectrometry experiments, for example, should be useful, if targeted at specific compounds, where interferences can be eliminated.

Further investigation of radical and $\mathrm{NO}_{\mathrm{xy}}$ budgets under conditions of significant organic aerosol concentration is required to determine the effect of heterogeneous reactions in these aromatic systems.

The possibility of an $\mathrm{OH}$ regeneration pathway, such as that discussed in Sect. 4.4.1, needs further investigation. Flash photolysis experiments with time-resolved $\mathrm{OH}$ detection by laser induced fluorescence could be carried out to determine radical yields in the initial stages of aromatic oxidation. The hydroxycyclohexadienyl radical, which in the atmosphere is formed by $\mathrm{OH}$ addition to the aromatic ring, could be generated by photolysis of a suitable precursor compound. If the postulated $\mathrm{OH}$ regeneration mechanism illustrated in Fig. 11 were correct, subsequent reactions with $\mathrm{O}_{2}$ and intramolecular rearrangements would lead to production of $\mathrm{OH}$ in the absence of NO. This experiment would require synthesis of a suitable photolytic precursor for the hydroxycyclohexadienyl radical, and careful consideration of the appropriate initial precursor and $\mathrm{O}_{2}$ concentrations. 
Acknowledgements. This work was supported by the European Commission within the EXACT project, Contract No. EVK2-CT1999-00053, and by the Ministerio de Ciencia y Tecnologia within Contract No. REN2000-3277-CE/CLI. The CEAM Foundation is supported by the Generalitat Valenciana and BANCAIXA.

Edited by: W. E. Asher

\section{References}

Arens, F., Gutzwiller, L., Baltensperger, U., Gäggeler, H. W., and Ammann, M.: Heterogeneous Reaction of $\mathrm{NO}_{2}$ on Diesel Soot Particles, Environ. Sci. Technol., 35, 2191-2199, 2001.

Atkinson, R. and Aschmann, S. M.: Products of the gas-phase reactions of aromatic hydrocarbons: Effect of $\mathrm{NO}_{2}$ concentration, Int. J. Chem. Kinet., 26, 929-944, 1994.

Bartolotti, L. J. and Edney, E. O.: Density-Functional Theory Derived Intermediates from the Oh Initiated Atmospheric Oxidation of Toluene, Chem. Phys. Lett., 245 (1), 119-122, 1995.

Bethel, H. L., Arey, J., and Atkinson, R.: Products of the $\mathrm{OH}$ radical-initiated reaction of 3-hexene-2,5-dione, Environ. Sci. Technol., 35 (22), 4477-4480, 2001.

Bierbach, A., Barnes, I., Becker, K. H., and Wiesen, E.: Atmospheric Chemistry of Unsaturated Carbonyls - Butenedial, 4-Oxo-2-Pentenal, 3-Hexene-2,5-Dione, Maleic-Anhydride, 3hFuran-2-One, and 5-Methyl-3h-Furan-2-One, Environ. Sci. Technol., 28 (4), 715-729, 1994.

Bloss, C., Wagner, V., Bonzanini, A., Jenkin, M. E., Wirtz, K., Martin-Reviejo, M., and Pilling, M. J.: Evaluation of detailed aromatic mechanisms (MCMv3 and MCMv3.1) against environmental chamber data, Atmos. Chem. Phys., 5, 623-639, 2005,

SRef-ID: 1680-7324/acp/2005-5-623.

Bloss, W. J., Lee, J. D., Bloss, C., Wirtz, K., Martin-Reviejo, M., Siese, M., Heard, D. E., and Pilling, M. J.: Validation of the calibration of a laser-induced fluorescence instrument for the measurement of $\mathrm{OH}$ radicals in the atmosphere, Atmos. Chem. Phys., 4, 571-583, 2003,

\section{SRef-ID: 1680-7324/acp/2004-4-571.}

Bröske, R., Kleffmann, J., and Wiesen, P.: Heterogeneous conversion of $\mathrm{NO}_{2}$ on secondary organic aerosol surfaces: A possible source of nitrous acid (HONO) in the atmosphere?, Atmos. Chem. Phys., 3, 469-474, 2003,

\section{SRef-ID: 1680-7324/acp/2003-3-469.}

Calvert, J. G., Atkinson, R., Becker, K. H., Kamens, R. M., Seinfeld, J. H., Wallington, T. J., and Yarwood, G.: The Mechanisms of Atmospheric Oxidation of Aromatic Hydrocarbons, Oxford University Press, 2002.

Derwent, R. G., Jenkin, M. E., and Saunders, S. M.: Photochemical ozone creation potentials for a large number of reactive hydrocarbons under European conditions, Atmos. Environ., 30 (2), 181-199, 1996.

Graedler, F. and Barnes, I.: Photolysis of Z-/E-3-Hexenedione. EUPHORE Annual Report 1997, 148, 1997.

Grosjean, E. and Grosjean, D.: The reaction of unsaturated aliphatic oxygenates with ozone, J. Atmos. Chem., 32 (2), 205-232, 1999.

Gutzwiller, L., Arens, F., Baltensperger, U., Gäggeler, H. W., and Ammann, M.: Significance of Semivolatile Diesel Exhaust Organics for Secondary HONO Formation, Environ. Sci. Technol., 36, 677-682, 2002.
Hamilton, J. F., Lewis, A. C., Bloss, C., Wagner, V., Henderson, A. P., Golding, B. T., Wirtz, K., Martin-Reviejo, M., and Pilling, M. J.: Measurements of photo-oxidation products from the reaction of a series of alkyl-benzenes with hydroxyl radicals during EXACT using comprehensive gas chromatography, Atmos. Chem. Phys., 3, 1999-2014, 2003,

SRef-ID: 1680-7324/acp/2003-3-1999.

Jenkin, M. E., Saunders, S. M., Derwent, R. G., and Pilling, M. J.: Construction and application of a master chemical mechanism (MCM) for modelling tropospheric chemistry, Abstr. Pap. Am. Chem. Soc., 214, 116-COLL, 1997.

Jenkin, M. E., Saunders, S. M., Wagner, V., and Pilling, M. J.: Protocol for the development of the Master Chemical Mechanism, MCM v3 (Part B): tropospheric degradation of aromatic volatile organic compounds, Atmos. Chem. Phys., 3, 181-193, 2003, SRef-ID: 1680-7324/acp/2003-3-181.

Kalberer, M., Paulsen, D., Sax, M., Steinbacher, M., Dommen, J., Prevot, A. S. H., Fisseha, R., Weingartner, E., Frankevich, V., Zenobi, R., and Baltensperger, U.: Identification of polymers as major components of atmospheric organic aerosols, Science, 303 (5664), 1659-1662, 2004.

Klotz, B., Volkamer, R., Hurley, M. D., Andersen, M. P. S., Nielsen, O. J., Barnes, I., Imamura, T., Wirtz, K., Becker, K. H., Platt, U., Wallington, T. J., and Washida, N.: OH-initiated oxidation of benzene - Part II. Influence of elevated NOx concentrations, Phys. Chem. Chem. Phys., 4 (18), 4399-4411, 2002.

Kwok, E. S. C., Aschmann, S. M., Atkinson, R., and Arey, J.: Products of the gas-phase reactions of o-, $\mathrm{m}$ - and $\mathrm{p}$-xylene with the $\mathrm{OH}$ radical in the presence and absence of $\mathrm{NO}_{\mathrm{x}}$, J. Chem. Soc.Faraday Trans., 93, 2847-2854, 1997.

Martin, P., Tuazon, E. C., Aschmann, S. M., Arey, J., and Atkinson, R.: Formation and atmospheric reactions of 4,5-dihydro-2methylfuran, J. Phys. Chem. A, 106 (47), 11 492-11 501, 2002.

Olariu, R.: Atmospheric Oxidation of Selected Aromatic Hydrocarbons, Doctoral Thesis, Bergische Universität Gesamthochschule Wuppertal, Wuppertal, 2001.

Olariu, R. I., Barnes, I., Becker, K. H., and Klotz, B.: Rate coefficients for the gas-phase reaction of $\mathrm{OH}$ radicals with selected dihydroxybenzenes and benzoquinones, Int. J. Chem. Kinet., 32 (11), 696-702, 2000.

Olariu, R. I., Klotz, B., Barnes, I., Becker, K. H., and Mocanu, R.: FT-IR study of the ring-retaining products from the reaction of $\mathrm{OH}$ radicals with phenol, o-, m-, and p-cresol, Atmos. Envir., 36 (22), 3685-3697, 2002.

Rea, G., Thuener, L. P., and Wenger, J.: Atmospheric Oxidation of Dimethylphenols, 8th European Symposium on PhysicoChemical Behaviour of Air Pollutants, Torino, Italy, 2001.

Saunders, S. M., Jenkin, M. E., Derwent, R. G., and Pilling, M. J.: Protocol for the development of the Master Chemical Mechanism, MCM v3 (Part A): tropospheric degradation of nonaromatic volatile organic compounds, Atmos. Chem. Phys., 3, 161-180, 2003,

SRef-ID: 1680-7324/acp/2003-3-161.

Smith, D. F., McIver, C. D., and Kleindienst, T. E.: Primary product distribution from the reaction of hydroxyl radicals with toluene at ppb NOX mixing ratios, J. Atmos. Chem., 30 (2), 209-228, 1998.

Thuener, L. P., Rea, G., and Wenger, J.: Photolysis of butenedial and 4-oxo-2-pentenal, EUPHORE Annual Report 2001, 2003 a. 
Volkamer, R.: A DOAS study on the oxidation mechanism of aromatic hydrocarbons under simulated atmospheric conditions, Dissertation, University of Heidelberg, Heidelberg, http://www. dissertation.de, 2001.

Volkamer, R., Platt, U., and Wirtz, K.: Primary and secondary glyoxal formation from aromatics: Experimental evidence for the bicycloalkyl-radical pathway from benzene, toluene, and pxylene, J. Phys. Chem. A, 105 (33), 7865-7874, 2001.

Volkamer, R., Klotz, B., Barnes, I., Imamura, T., Wirtz, K., Washida, N., Becker, K. H., and Platt, U.: OH-initiated oxidation of benzene - Part I. Phenol formation under atmospheric conditions, Phys. Chem. Chem. Phys., 4 (9), 1598-1610, 2002.
Wagner, V., Jenkin, M. E., Saunders, S. M., Stanton, J., Wirtz, K., and Pilling, M. J.: Modelling of the photooxidation of Toluene: conceptual ideas for validating detailed mechanisms, Atmos. Chem. Phys., 3, 89-106, 2003,

SRef-ID: 1680-7324/acp/2003-3-89.

Yu, J. Z. and Jeffries, H. E.: Atmospheric photooxidation of alkylbenzenes 2. Evidence of formation of epoxide intermediates, Atmos. Envir., 31, 2281-2287, 1997. 